\title{
Modulating Dopamine Signaling and Behavior with Chemogenetics: Concepts, Progress, and Challenges
}

\author{
Annika Højrup Runegaard, Ciarán Martin Fitzpatrick, David Paul Drucker Woldbye, Jesper Tobias Andreasen, \\ Andreas Toft Sørensen, and Ulrik Gether
}

Molecular Neuropharmacology and Genetics Laboratory, Department of Neuroscience (A.H.R., D.P.D.W., A.T.S., U.G.) and Department of Drug Design and Pharmacology (C.M.F., J.T.A.), Faculty of Health and Medical Sciences, University of Copenhagen, Copenhagen, Denmark

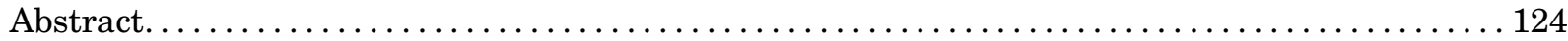

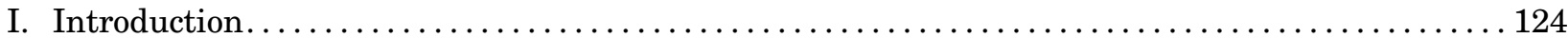

A. A Portrait of the Dopamine System .................................. 125

1. Dopamine Synthesis and Neurotransmission ........................... 125

2. Dopamine Pathways and Circuits................................. 126

B. Chemogenetics-Pharmacological Remote Control of Neuronal Activity ............... 126

1. Specific Spatial Resolution of Designer Receptors Exclusively Activated by Designer

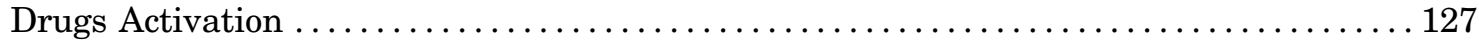

2. Specific Temporal Resolution of Designer Receptors Exclusively Activated by Designer Drugs Activation....................................... 129

II. Chemogenetic Approaches for Dissecting Dopamine-Mediated Behaviors. ............... 130

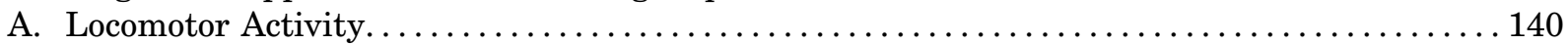

1. Dopamine Activity Correlates with Locomotor Activity_Proof-of-Concept Studies . . . . 140

2. The Relative Output-Activity from Direct Medium Spiny Neurons and Indirect Medium Spiny Neurons Determines Locomotor Activity ...................... 140

3. Locomotor Activity_A Simple Behavior with Multiple Levels of Regulation........... 141

4. Dysfunctional Motor Control in Parkinson's Disease............................ 142

B. Motivation and Reward-Guided Behaviors................................. 143

1. Substantiating Dopamine as a Key Regulator of Motivation ................... 143

2. The Role of Direct Medium Spiny Neurons and Indirect Medium Spiny Neurons in Regulating Reward-Motivated Behaviors............................... 143

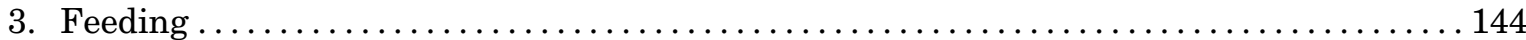

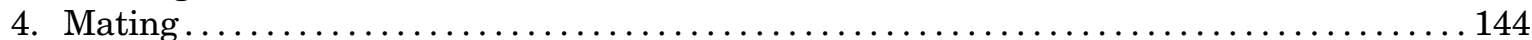

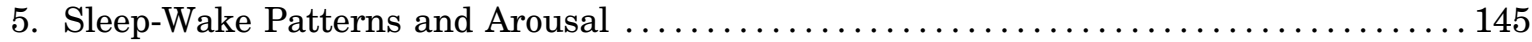

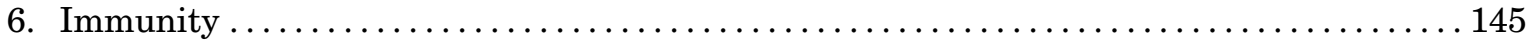

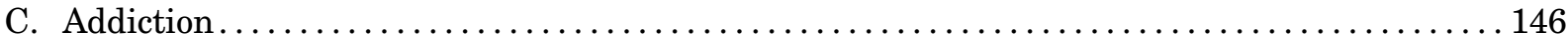

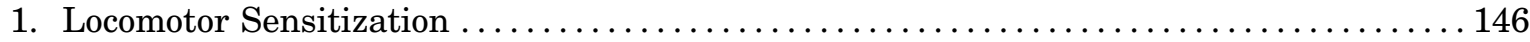

2. Reward Perception, Drug-Cue Associations, and Relapse Behaviors............... 146

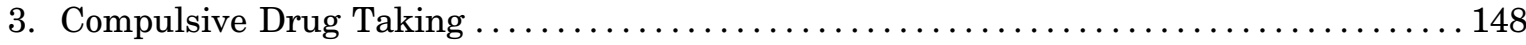

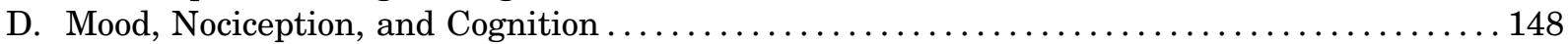

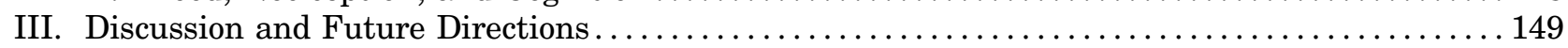

A. Designer Receptors Exclusively Activated By Designer Drugs Effects in Theory and in

Reality ....................................................... 149

Address correspondence to: Ulrik Gether, Molecular Neuropharmacology and Genetics Laboratory, Department of Neuroscience, University of Copenhagen, Mærsk Tower 5th floor, 3 Blegdamsvej, DK-2200, Copenhagen, Denmark. E-mail: gether@sund.ku.dk; or Andreas Toft Sørensen, Molecular Neuropharmacology and Genetics Laboratory, Department of Neuroscience, University of Copenhagen, Mærsk Tower 5th floor, 3 Blegdamsvej, DK-2200, Copenhagen, Denmark. E-mail: andreass@sund.ku.dk

A.H.R. and C.M.F. contributed equally as first authors.

A.T.S. and U.G. contributed equally as corresponding authors.

This work was supported by the UCPH 2016 Program of Excellence and the Lundbeck Foundation.

https://doi.org/10.1124/pr.117.013995. 
1. Designer Receptors Exclusively Activated By Designer Drugs Expression-The

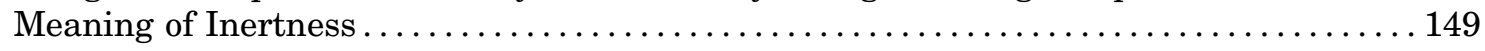

2. Interpretation of Activating Designer Receptors Exclusively Activated By Designer Drugs with Clozapine N-Oxide.................................. 150

3. Considerations for Repeated Activation of Designer Receptors Exclusively Activated

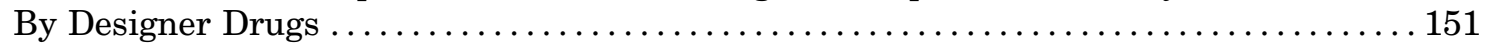

4. Can Selective Dopamine Expression Be Achieved? ........................ 151

5. Can Designer Receptors Exclusively Activated By Designer Drugs Help Dissect the Dopaminergic Pathways? ......................................... 151

B. Have Chemogenetic Studies Advanced Our Understanding of Dopamine-Related Behaviors? ...................................................... 152

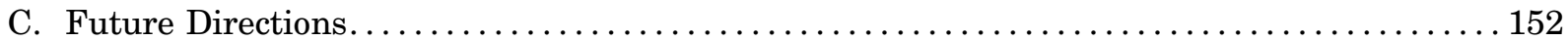

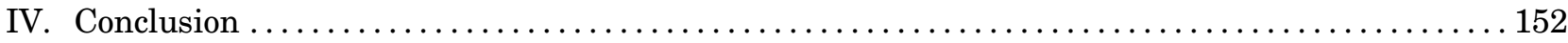

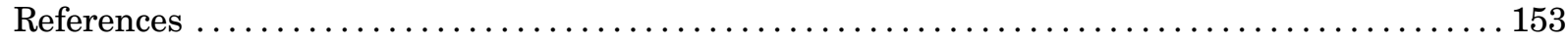

\begin{abstract}
For more than 60 years, dopamine (DA) has been known as a critical modulatory neurotransmitter regulating locomotion, reward-based motivation, and endocrine functions. Disturbances in DA signaling have been linked to an array of different neurologic and psychiatric disorders, including Parkinson's disease, schizophrenia, and addiction, but the underlying pathologic mechanisms have never been fully elucidated. One major obstacle limiting interpretation of standard pharmacological and transgenic interventions is the complexity of the DA system, which only appears to widen as research progresses. Nonetheless, development of new genetic tools, such as chemogenetics, has led to an entirely new era for functional studies of neuronal signaling. By exploiting receptors that are engineered to respond selectively to an otherwise inert ligand, so-called Designer Receptors Exclusively Activated by
\end{abstract}

Designer Drugs (DREADDs), chemogenetics enables pharmacological remote control of neuronal activity. Here we review the recent, extensive application of this technique to the DA field and how its use has advanced the study of the DA system and contributed to our general understanding of DA signaling and related behaviors. Moreover, we discuss the challenges and pitfalls associated with the chemogenetic technology, such as the metabolism of the DREADD ligand clozapine $\mathrm{N}$-oxide (CNO) to the D2 receptor antagonist clozapine. We conclude that despite the recent concerns regarding CNO, the chemogenetic toolbox provides an exceptional approach to study neuronal function. The huge potential should promote continued investigations and additional refinements to further expound key mechanisms of DA signaling and circuitries in normal as well as maladaptive behaviors.

\section{Introduction}

Pioneering studies showed more than 60 years ago that dopamine (DA) is not just a metabolic intermediate in the synthesis of norepinephrine but a neurotransmitter in itself (Carlsson and Waldeck, 1958). The DA system has since been the subject of numerous studies, and it is now fully established that DA is an essential and dominant neurotransmitter within the central nervous system (CNS), modulating a wide array of physiologic functions, such as motor control, rewardbased motivation, memory, learning, decision making, and neuroendocrine secretion (Björklund and Dunnett, 2007; Berke, 2018). The DA field has been further fostered by the realization that imbalances in DA homeostasis are critical components of both neurologic and psychiatric diseases such as Parkinson's disease (PD), schizophrenia, bipolar disorder, Tourette's syndrome, attention-deficit/hyperactivity disorder (ADHD), and addiction (Hornykiewicz, 1966; Beaulieu and Gainetdinov, 2011; Tritsch and Sabatini, 2012).

A major challenge has been to dissect the molecular and cellular mechanisms underlying the ability of DA to regulate a broad spectrum of brain circuits and thereby influence multiple physiologic and pathophysiological processes in the CNS. One main reason is the complexity and heterogeneity of the DA system, which has become even more apparent as research has progressed over the years. As a consequence, multiple aspects of DA function still appear remarkably poorly understood. This situation has called for the implementation of

ABBREVIATIONS: AAV, adeno-associated virus; AC, adenylyl cyclase; ADHD, attention-deficit/hyperactivity disorder; BNST, bed nucleus of the stria terminalis; CAV2, canine adenovirus type 2; CNO, clozapine $N$-oxide; CNS, central nervous system; CPP, conditioned place preference; $\mathrm{D}_{1-5} \mathrm{R}$, dopamine receptors 1-5; DA, dopamine; DAT, dopamine transporter; DIO, double-floxed inverse open reading frame; dMSN, direct medium spiny neuron; DREADDs, designer receptors exclusively activated by designer drugs; ERK, extracellular signalregulated kinase; Flp, flippase; GIRK, G-protein coupled inward-rectifying potassium channels; Gi/q/s, regulatory G-protein subunit; GPCR, G protein-coupled receptor; hM3Dq, Gq DREADD; hM4Di, Gi DREADD; 5-HT, 5-hydroxytryptamine; iMSN, indirect medium spiny neuron; KO, knockout; KORD, kappa-opioid receptor-DREADD; L-DOPA, L-3,4-dihydroxyphenylalanine; MSN, medium spiny neuron; NAc, nucleus accumbens; 6-OHDA, 6-hydroxy-dopamine; PD, Parkinson's disease; PFC, prefrontal cortex; PKA, protein kinase A; rM3Darr, beta-arrestin based DREADD; rM3Ds, Gs DREADD; SalB, salvinorin B; SN, substantia nigra; TH, tyrosine hydroxylase; VTA, ventral tegmental area; WT, wild type. 
novel techniques in the field encompassing particular tools that can harness specific manipulations of neuronal circuits to elucidate mechanisms of homeostatic and pathologic phenotypes. The advancements of transformative molecular-genetic manipulation technologies, such as chemogenetics and optogenetics, are altering the course of behavioral neuroscience with the potential to unprecedentedly advance our knowledge about how DAergic signaling and its complex organization mediates behavior. It is therefore of no surprise that these technologies already have been widely used as strategies to study and dissect DA-related functions. Despite their many obvious advantages, such techniques inevitably also embrace a substantial number of pitfalls and limitations that must be considered when interpreting experimental results.

In this review, we carry out a comprehensive review of studies over the last few years of the DA system in which chemogenetic approaches have been employed; that is, studies, that have taken advantage of DREADDs ("Designer Receptors Exclusively Activated by Designer Drugs") and thereby used the possibility of activating discrete $\mathrm{G}$ protein-coupled signaling cascades in selected target cells to manipulate neuronal functions. We believe that the technique possesses huge potential and can be a powerful approach if used in the appropriate manner. We will provide a short overview of the DA system before introducing the chemogenetic approach and critically reviewing areas of DA research in which chemogenetic approaches have been applied. Next, we will specifically describe pitfalls and limitations, as well as discuss future directions for refining the technology. We hope thereby to generate a detailed overview of what we have learned from the use of chemogenetics and a framework for how chemogenetics can fruitfully be applied to the DA system to continue the exploration of this fascinating neurotransmitter.

\section{A. A Portrait of the Dopamine System}

\section{Dopamine Synthesis and Neurotransmission.} DA neurotransmission depends on DA synthesis that takes place within a restricted set of neurons expressing the enzymes involved in the biosynthetic pathway of DA from dietary tyrosine sources. Tyrosine hydroxylase (TH), the rate-limiting enzyme in DA production, converts tyrosine to L-3,4-dihydroxyphenylalanine (L-DOPA), which in turn is converted by aromatic amino acids decarboxylase to DA. Cytosolic DA is sequestered into synaptic vesicles by the action of the vesicular monoamine transporter 2 , acting as a proton antiporter that takes advantage of the low intravesicular $\mathrm{pH}$ to transport DA into the vesicles against a large concentration gradient (German et al., 2015). After fusion of these vesicles with the plasma membrane and release from presynaptic terminals, DA exerts its actions via five distinct, but highly homologous, $\mathrm{G}$ protein-coupled receptors (GPCRs). The DA receptors are divided into two subclasses based on their pharmacological profile and coupling: the D1-like receptors $\left(D_{1} R\right.$ and $\left.D_{5} R\right)$ and the D2-like receptors $\left(\mathrm{D}_{2} \mathrm{R}, \mathrm{D}_{3} \mathrm{R}\right.$, and $\mathrm{D}_{4} \mathrm{R}$ ) (Beaulieu and Gainetdinov, 2011). The $\mathrm{D}_{2} \mathrm{R}$ exists in a short or long variant $\left(\mathrm{D}_{2} \mathrm{~S}\right.$ and $\left.\mathrm{D}_{2} \mathrm{~L}\right)$ of which $\mathrm{D}_{2} \mathrm{~S}$ constitutes the primary presynaptic autoreceptor in the DAergic neurons (Lindgren et al., 2003). D1-like receptors are generally characterized by displaying one to two orders of magnitude lower affinity to DA compared with the D2-like receptors, meaning that the latter are much more likely to be saturated with DA during tonic release conditions (Richfield et al., 1987). The coupling of the D1-like and D2-like receptors to intracellular signaling pathways are distinct and generally oppositely directed. D1-like receptors couple to $G_{\alpha \mathrm{s}}$ and $G_{\alpha o l f}$, leading to stimulation of adenylyl cyclase (AC), which results in increased levels of cAMP and activation of protein kinase $\mathrm{A}$ (PKA). In contrast, D2-like receptors couple to $\mathrm{G}_{\alpha \mathrm{i}}$ and $\mathrm{G}_{\alpha \mathrm{o}}$, leading to inhibition of AC, lower cAMP levels, and less PKA activation. Via the $\mathrm{G}_{\beta \gamma}$ subunits of the heterotrimeric $\mathrm{G}$ protein, the D2-like receptors can also regulate ion channel function, including $\mathrm{G}$ proteincoupled inward-rectifying potassium channels (GIRK) (Kuzhikandathil et al., 1998; Lavine et al., 2002) and voltage-gated $\mathrm{Ca}^{2+}$-channels (Yan et al., 1997). DA receptors, and in particular D2-like receptors, also signal through $\mathrm{G}$ protein-independent pathways. The D2-like receptors can, for example, operate through $\beta$-arrestin-dependent pathways involving activation of Akt (also called protein kinase B) and glycogen synthase kinase 3 (Beaulieu et al., 2004, 2005, 2007). For further details on DA receptors, including a more in-depth description of their functional coupling and distribution in the brain, we kindly refer to other reviews (Neve et al., 2004; Beaulieu and Gainetdinov, 2011; Tritsch and Sabatini, 2012; Beaulieu et al., 2015).

To understand the complex regulation of DA signaling, it is essential to highlight the DA transporter (DAT). DAT is expressed in DA neurons and mediates rapid reuptake of released DA. In doing so, DAT plays an essential role in controlling extracellular levels of DA and thereby DA signaling (Jones et al., 1998). DAT belongs to the large family of neurotransmitter:sodium symporters, and thus the transporter utilizes the transmembrane sodium gradient to translocate effectively DA across the membrane against its concentration gradient. Importantly, DAT is the prime target for the stimulatory action of psychostimulants such as cocaine and amphetamine. Whereas cocaine acts as a highaffinity, competitive inhibitor, amphetamine is a substrate that upon entry through the transporter promotes the release of DA via DAT, a process known to involve activation of protein kinases, such as $\mathrm{Ca}^{2+} /$ calmodulindependent protein kinase II and phosphorylation of the transporter itself (Kristensen et al., 2011). DAT is also a target for commonly used mediation used against ADHD such as methylphenidate and modafinil. For further 
details on DAT, we kindly refer to other excellent reviews (Torres and Amara, 2007; Kristensen et al., 2011; Sulzer, 2011).

2. Dopamine Pathways and Circuits. The majority of the brain's DAergic transmission is organized into four neural pathways that we now know partake in distinct, but also sometimes seemingly overlapping behaviors. 1) The nigrostriatal pathway projects from the midbrain substantia nigra (SN) pars compacta to the dorsal striatum and exerts both facilitatory and inhibitory regulation of movements and is involved in habitual and compulsive responses. 2) The mesolimbic pathway projects from the ventral tegmental area (VTA) to limbic brain regions, including the amygdala and the nucleus accumbens (NAc) of the ventral striatum. This pathway is involved in motivation, goal-directed behaviors, attributing incentive values of reward-related environmental stimuli and labeling pleasurable emotions with motivational salience. 3) The mesocortical pathway projects from the midbrain VTA to the prefrontal cortex (PFC) and chiefly mediates executive function such as selective and sustained attention, flexibleresponding to stimuli, planning and goal-directed behavior, and monitoring and inhibitory control. 4) The tuberoinfundibular pathway projects from the hypothalamus to the pituitary gland, where DA inhibits the secretion of the hormone prolactin (Volkow et al., 2007; Arias-Carrión and Pŏppel, 2007; Björklund and Dunnett, 2007).

Although the separation of the DA system into the above-mentioned pathways is still relevant, it is important to emphasize that recent investigations have led to a significantly more complicated picture of the DAergic system with heterogeneous populations of neurons that express and release DA via different mechanisms in different brain regions (Morales and Margolis, 2017). For example, labeling studies have revealed a complex heterogeneity of the midbrain DAergic system in terms of projection patterns, firing and release probability, and intracellular molecular characteristics and properties (Lammel et al., 2008, 2014; Apuschkin et al., 2015; Poulin et al., 2018). Furthermore, it has been demonstrated that some midbrain DA neurons are capable of releasing other neurotransmitters, such as 5-hydroxytryptamine (5-HT) (Zhou et al., 2005), glutamate (Chuhma et al., 2004; Nair-Roberts et al., 2008; Hnasko et al., 2010; Stuber et al., 2010), and GABA (Tritsch et al., 2014; Kim et al., 2015; Berrios et al., 2016). Finally, complex input networks projecting onto DAergic neurons, as well as output target areas (e.g., Beier et al., 2015; Poulin et al., 2018), emphasize a major role of DA as a modulator rather than a regulator of neuronal excitability in target areas. Once DA is released in target areas, DA receptors expressed on postsynaptic membranes convey and determine the further processing of the DAergic signal, which is dependent on the DA receptor profile. While target areas such as the dorsal striatum, comprised mainly of GABAergic medium spiny neurons (MSNs), have been found to be rather ordered in terms of expressing either D1- or D2-like receptors and with distinct projection patterns, other target areas such as the NAc and PFC lack such architecture or remain to be equally characterized (Beaulieu and Gainetdinov, 2011; Kupchik et al., 2015). Altogether, the DA system displays an anatomic, as well as molecular, complexity, which precludes standard pharmacologic or genetic approaches for deconstructing DA signaling in normal and maladaptive behavioral states. However, these obstacles can be circumvented if DAergic pathways are precisely defined and/or DAergic signaling can be both spatially and temporally controlled. Furthermore, if maladaptive DA states are reversible, a technique that would allow for bidirectional regulation would be a huge advantage for exploring new therapeutic avenues.

\section{B. Chemogenetics-Pharmacological Remote Control of Neuronal Activity}

The DREADD technology was developed by Bryan Roth and colleagues at the University of North Carolina and was published for the first time in 2007 (Armbruster et al., 2007). This methodological approach extends from other chemogenetic platforms described in the field of GPCRs, such as allele-specific activation of genetically encoded receptors (Strader et al., 1991), receptors activated solely by synthetic ligands (Conklin et al., 2008) and neoceptors (Jacobson et al., 2001). The development of DREADDs enabled new study designs as it allowed neuroscientists to bidirectionally manipulate intracellular G protein-coupled signaling of neuronal populations of interest and at sufficient strength-acting like a device for remote control of neuronal signaling. We will briefly describe the rationale behind the DREADDs technique and elaborate on its suitability for studying the DA system. For more information on DREADDs development and structures, see Roth (2016) and Armbruster et al. (2007).

The original DREADDs are mutated muscarinic acetylcholine receptors, which are insensitive to their natural ligand, acetylcholine, and instead respond to an otherwise inert compound, clozapine $N$-oxide (CNO), a metabolite of the atypical antipsychotic clozapine (Roth, 2016). The basic idea was to genetically introduce and express the various DREADD variants within cells of interest and thereby allow targeted manipulation of specific intracellular signaling cascades through the coupling of the distinct receptors to either excitatory ( $\mathrm{Gs}, \mathrm{Gq}$ ) or inhibitory ( $\mathrm{Gi}$ ) signaling cascades upon CNO binding (see Fig. 1). Specifically, the Gq-coupled hM3Dq DREADD leads to activation of the phospholipase C cascade and increases intracellular calcium to promote neuronal burst firing (Armbruster et al., 2007), while the Gs-coupled rM3Ds DREADD increases cAMPmediated depolarization and thus mimics the signaling 


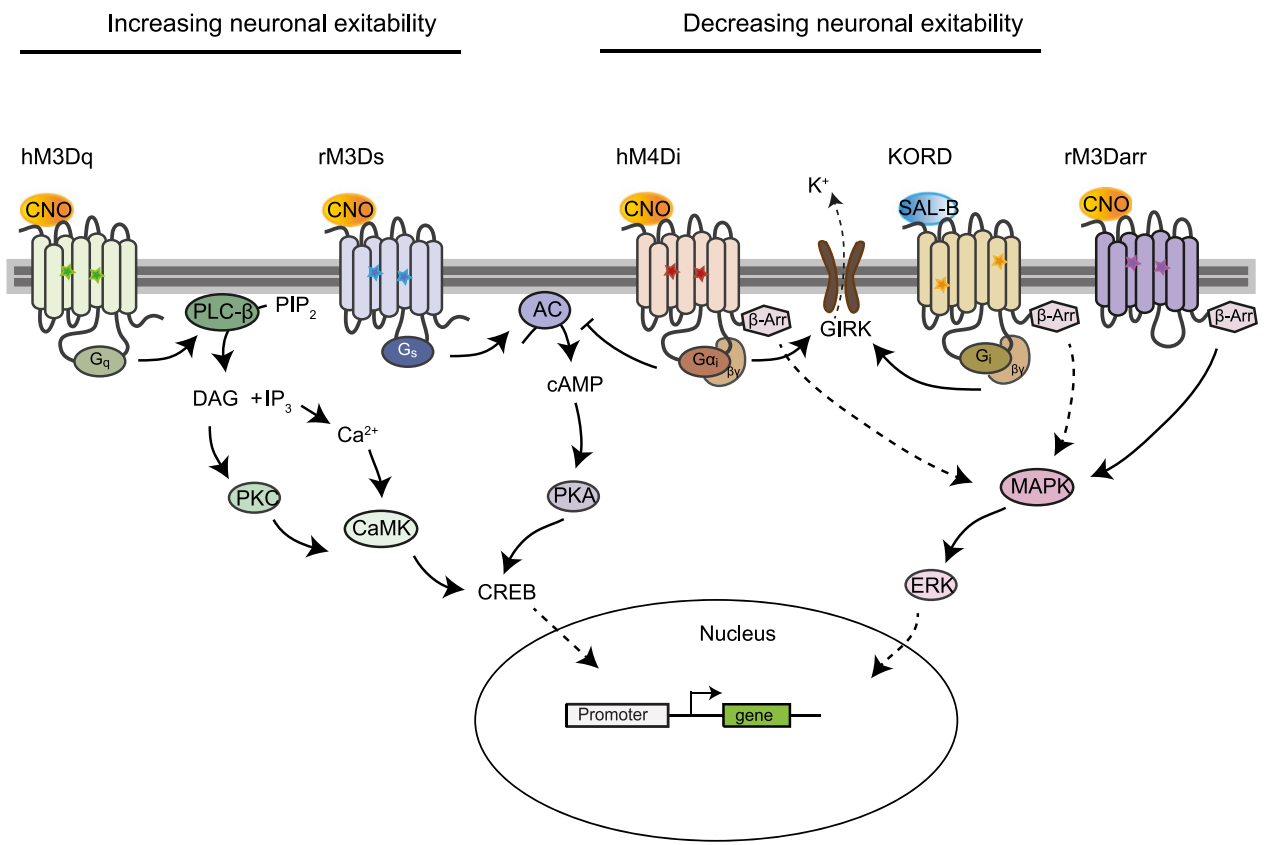

Fig. 1. The chemogenetic toolbox contains a variety of engineered receptors (i.e., DREADDs and the KORD) that upon activation, via CNO or SalB, initiate endogenous signaling cascades and regulate canonical signaling and excitability (Roth 2016; Armbruster et al., 2007; Vardy et al., 2015). Four of the engineered receptors are scaffolded on the muscarinic acetylcholine receptor, respond to CNO, and couple to Gq (hM3Dq), Gs (rM3Ds), Gi (hM4Di) or $\beta$-arrestin (rM3Darr). KORD is scaffolded on the kappa-opioid receptor and responds to SalB, but signal as hM4Di via the Gi-coupled pathway. However, the kinetic profile of the KORD-SalB is different from hM4Di-CNO and provides a faster onset of action. While hM3Dq and rM3Ds increase neuronal excitability by activating the PKC and PKA pathways, respectively, hM4Di and KORD decrease neuronal excitability by activating GIRK channels and inhibiting the PKA pathway. The beta-arrestin biased rM3Darr initiates beta-arrestin signaling, which distinguishes the latestage signaling pathway from Gi-coupled receptors signaling via mitogen-activated protein kinase/ERK.

pathway activated by D1-like receptors (Farrell et al., 2013). The Gi-coupled hM4Di DREADD causes membrane hyperpolarization through activation of GIRK and decreases cAMP signaling (Armbruster et al., 2007) analogous to $D_{2} R s$. Like other GPCRs, activated DREADDs may recruit arrestins, which are able to convey delayed $G$ protein-independent receptor signaling (Roth, 2016). A beta-arrestin based DREADD (rM3Darr) has been generated to explore the physiologic relevance of these arrestin-dependent signaling pathways, but overall this receptor has been used much less than the other aforementioned variants (Nakajima and Wess, 2012; Roth, 2016). Finally, a new DREADD variant, the kappa-opioid receptor DREADD (KORD), constitutes a mutated kappaopioid receptor that exerts inhibitory actions via a Gi-coupled signaling pathway. This receptor is activated by salvinorin B (SalB), an inert metabolite of the psychotropic salvinorin A (Vardy et al., 2015). This new variant can be used alone, as well as opening up the possibility for combinatorial DREADD studies, where KORD can be applied together with CNOsensitive DREADDs. Of further interest, the pharmacokinetics of KORD activation by SalB differs greatly from that of CNO-sensitive DREADDs, as it possesses a much more rapid onset of action and response extinction timeframe within 1 hour in contrast to several hours for the effects of CNO (Vardy et al., 2015).
1. Specific Spatial Resolution of Designer Receptors Exclusively Activated by Designer Drugs Activation. A major advantage of chemogenetics, compared with classic pharmacological manipulations, is the specificity by which activation of the receptors can be achieved. The spatial resolution of DREADD-induced manipulations is determined by the ectopic expression of DREADDs in desired neurons, and this can in principle be controlled by the researcher's choice of approach: either directly by placing the DREADD transgene downstream of a specific promoter in transgenic animals or virus vectors (Farrell et al., 2013; Ferguson et al., 2013) or indirectly through Cre-lox-mediated recombination using double-floxed inverse open reading frame (DIO) DREADD-encoding viruses in combination with rodent Cre-driver lines or a second Cre-expressing virus (Bock et al., 2013; Carter et al., 2013; Schwarz et al., 2015). Figure 2 demonstrates how various viraltransgenic approaches can be applied to target midbrain DA neurons and here exemplified how to obtain gradually better selective expression toward only NAcprojecting neurons. While viral delivery of a DREADD transgene may limit expression to the target area e.g., VTA (Fig. 2A), the DREADD transgene could be introduced downstream of promoters, regulating genes that are selectively expressed within the DA system to obtain more desirable expression. Such an approach has been applied selectively to target DREADDs in dorsostriatal MSNs belonging to either the striatonigral direct 
A Single viral approach in WT mice

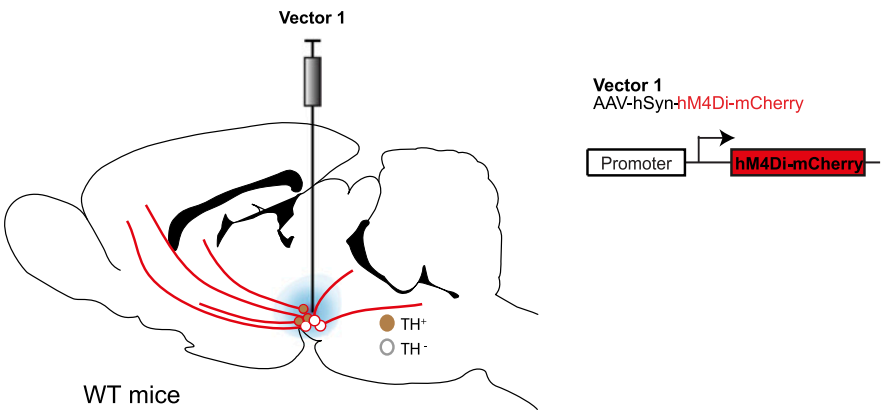

B Single viral Cre-dependent approach in Cre-driver lines

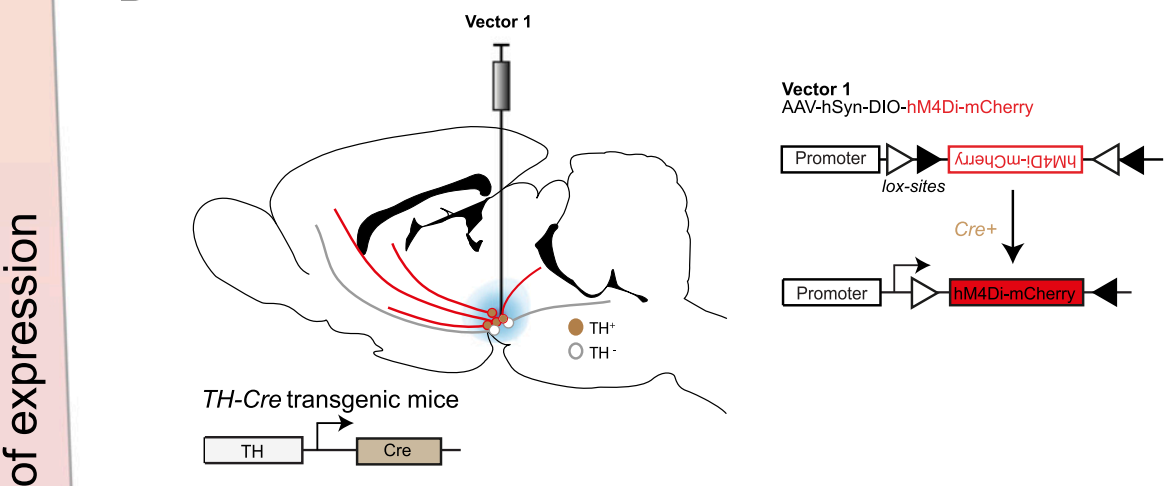

C Dual viral Cre-dependent approach in WT mice

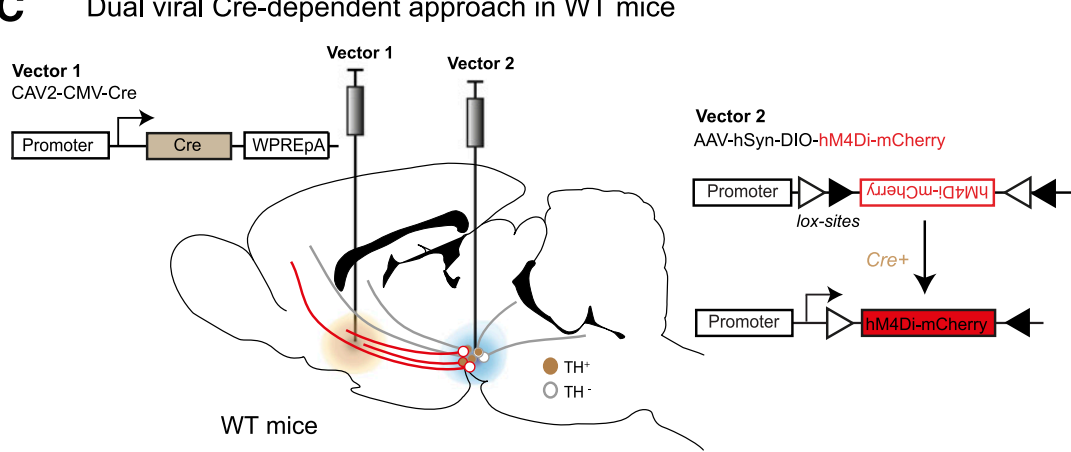

D Dual viral Flp- and Cre-dependent approach in Cre-driver lines

Vector 1

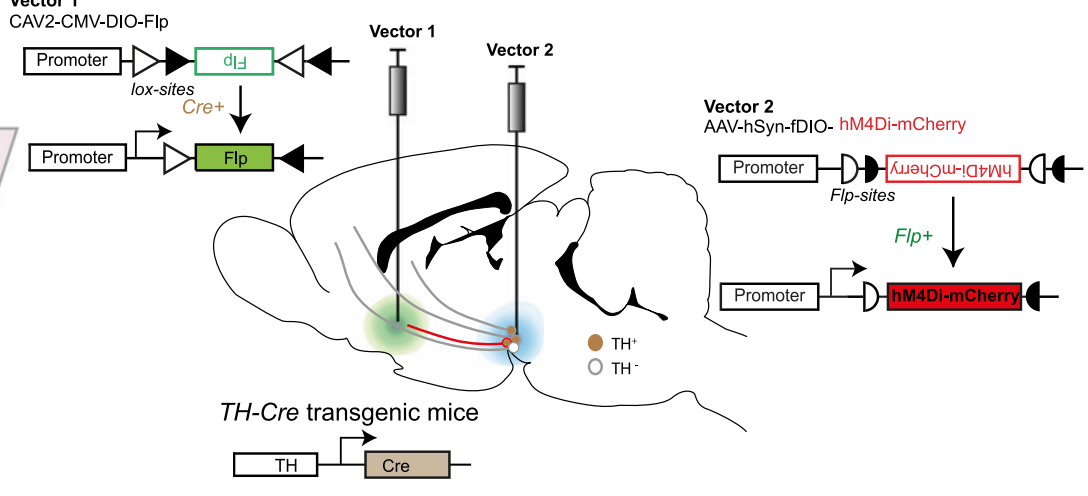

Fig. 2. Viral approaches to obtain increased specificity of chemogenetic manipulations within the DA system. (A-D) Illustrates different viraltransgenic approaches to obtain chemogenetic control of midbrain DA neurons with increasing specificity of DREADD expression toward NAcprojecting neurons (shown for hM4Di-mCherry). (A) The viral approach that limits DREADD expression primarily toward the injection site; a viral vector carrying the DREADD transgene is injected into the target area of a WT mouse. Increased selectivity can be obtained by placing the DREADD transgene downstream of a neuron-specific promoter (e.g., Ferguson et al., 2011; Farrell et al., 2013; Ferguson et al., 2013). (B) The viral Cre-dependent approach is by far the most commonly used to obtain chemogenetic control of a neuronal population of interest; a single Cre-dependent DREADD vector is injected into the target area of a Cre-driver mouse and restricts the expression toward Cre-expressing neurons within the injection site (e.g., Lindeberg et al., 2004; Savitt et al., 2005; Bäckman et al., 2006; Runegaard et al., 2018). (C) The dual viral Cre-dependent approach has been applied to 
pathway (i.e., $\mathrm{D}_{1} \mathrm{R}$ expressing) or the striatopallidal indirect pathway (i.e., $\mathrm{D}_{2} \mathrm{R}$ expressing) by using herpes simplex virus carrying dynorphin and enkephalin promoters, respectively (Ferguson et al., 2011). Targeting of midbrain DA neurons has been successful using a combinatorial approach, with the most commonly applied approach taking advantage of transgenic Cre-driver lines (Fig. 2B). More specifically, injection of a viral vector (e.g., AAV), carrying a DIO-DREADD (the expression of which relies on the presence of Cre), into the target area (e.g., VTA) of Cre-driver lines exploiting classic genetic markers of DA neurons, confines DREADD expression to putative DA neurons in the midbrain. Classic markers include the DA transporter (DAT) and tyrosine hydroxylase (TH), and both DAT-Cre and TH-Cre driver lines have been used to confine DREADD expression to midbrain DA neurons (Lindeberg et al., 2004; Savitt et al., 2005; Bäckman et al., 2006; Runegaard et al., 2018) (Fig. 2B). Alternatively, the Cre-recombinase can be delivered by viral means in a dual viral approach in wild-type (WT) mice (Fig. 2C), as recently demonstrated by use of retrogradely transported recombinant canine adenovirus (CAV2) (Hnasko et al., 2006; Boender et al., 2014; Boekhoudt et al., 2016) or retrograde adeno-associated virus (AAV2-retro) (Tervo et al., 2016). The dual viral approach can even provide extra target specificity toward individual DA projections in Cre-driver lines. In such a setup, combinatorial viral expression determined by a retrograde vector encoding Cre-dependent recombinase flippase (Flp) and a second viral vector encoding Flp-dependent DREADD can confine the expression further (Fig. 2D) (Boender et al., 2014; Runegaard et al., 2018). In addition, although less well characterized for achieving high DA neuron specificity, an AAV vector delivering Cre-recombinase under the control of a fragment of the TH promoter is another option (Stauffer et al., 2016). With a new delivery platform allowing for intravenous AAV delivery, it appears that DA neurons can even be selectively targeted by systemic application (Chan et al., 2017). These two latter viral approaches are interesting, as they technically permit DREADD targeting toward DA neurons in species other than rodents (Stauffer et al., 2016). Taken together, the molecular-genetic toolbox for chemogenetics is large and ideally suited for targeting and studying the DA system (Whissell et al., 2016). Since the signaling cascade of
DREADDs and DA receptors are conveyed through the same downstream molecules, it is also noteworthy to mention that activation of DREADDs possesses the potential ability to mimic, mitigate, or override endogenous DA signaling.

2. Specific Temporal Resolution of Designer Receptors Exclusively Activated by Designer Drugs Activation. The temporal resolution of DREADDs activation represents another important variable. Indeed, as mentioned above, the DREADD and KORD agonists, CNO and SalB, respectively, display distinct temporal dynamics (Roth, 2016). Furthermore, the route of administration can strongly influence the pharmacokinetics and thereby the temporal dynamics of the manipulation. Systemic administration of CNO by intraperitoneal injection causes plasma levels to peak within $30 \mathrm{~min}$ and decline over the subsequent $2 \mathrm{~h}$ (Guettier et al., 2009). Although plasma levels of CNO decline quickly, behavioral effects may be longer lasting for up to $6-10 \mathrm{~h}$ (Alexander et al., 2009; Wang et al., 2013). It is likely that this is linked to the recently reported possible conversion of CNO to clozapine following in vivo administration in rodents (Maclaren et al., 2016; Gomez et al., 2017). Strikingly, it appears that clozapine, but not $\mathrm{CNO}$, crosses the blood-brain barrier, and clozapine binds to and activates DREADD receptors with higher affinity and potency than CNO (Gomez et al., 2017). As clozapine is a $D_{2} R$ antagonist, this underscores the importance of including CNO-treated non-DREADDexpressing animals as controls, particularly in studies focusing on the DA system. Along these lines, it should be mentioned that the resulting concentrations of clozapine, however, are predicted to be too low to affect $\mathrm{D}_{2} \mathrm{R}$ function at the most commonly used concentrations of CNO in behavioral studies (Gomez et al., 2017; Mahler and Aston-Jones, 2018). Nonetheless, these findings support the use of subthreshold doses of clozapine as the DREADD-activating agent instead of high doses of CNO (Gomez et al., 2017). In contrast to $\mathrm{CNO}$, the pharmacokinetics of SalB have not been equally well studied, but behavioral effects after intraperitoneal administration of SalB indicates a 10-15 min onset of action time and roughly $1 \mathrm{~h}$ duration depending on the behavior investigated, thus providing both a

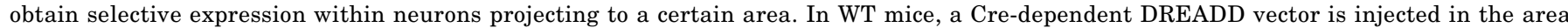

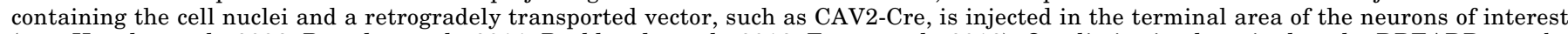

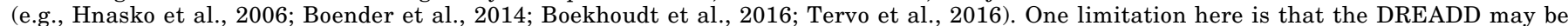

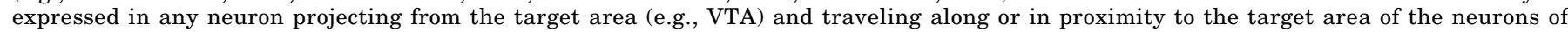

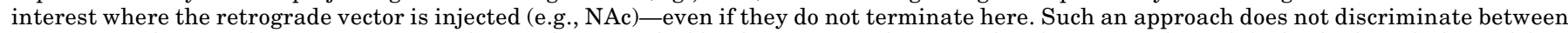

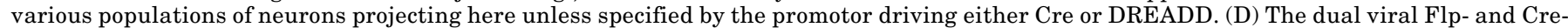

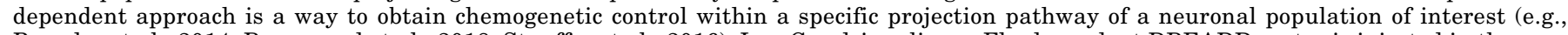

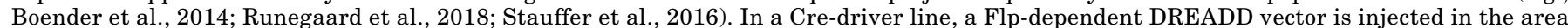

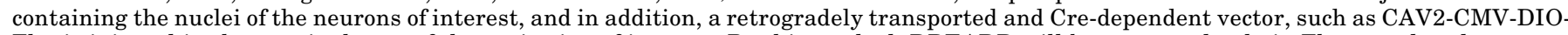

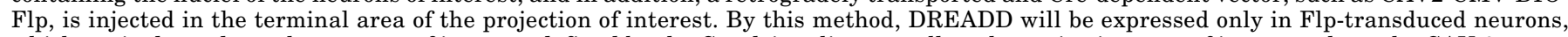

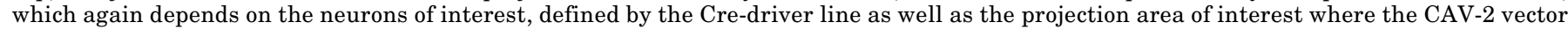
is injected.
} 
faster onset and elimination of its effect compared with CNO (Vardy et al., 2015; Marchant, et al., 2016b).

\section{Chemogenetic Approaches for Dissecting Dopamine-Mediated Behaviors}

In the following sections, we will review how the DREADD technology has been applied to investigate DA circuitries and signaling pathways in an array of different behaviors and pathologic states, including locomotion, PD, reward-motivated behaviors, addiction, and mood disorders. Figure 3 shows an anatomic overview of selected DA-related studies where the
DREADD technology has been employed and which approaches those that have been used to target selective regions, neurons, or projections of the DA system. A more extensive and elaborate overview of all DA-related chemogenetic studies published so far (77 papers in total), covering the period from 2011 till June 2018, is provided in Table 1, including information regarding area of research, test model, chemogenetic vector, target area/cell, strain, and major findings for easy comparison between studies. Considering that DREADDs have demonstrated their usefulness for studying neurotransmitter systems other than DA, we refer to excellent recent reviews covering a broader use

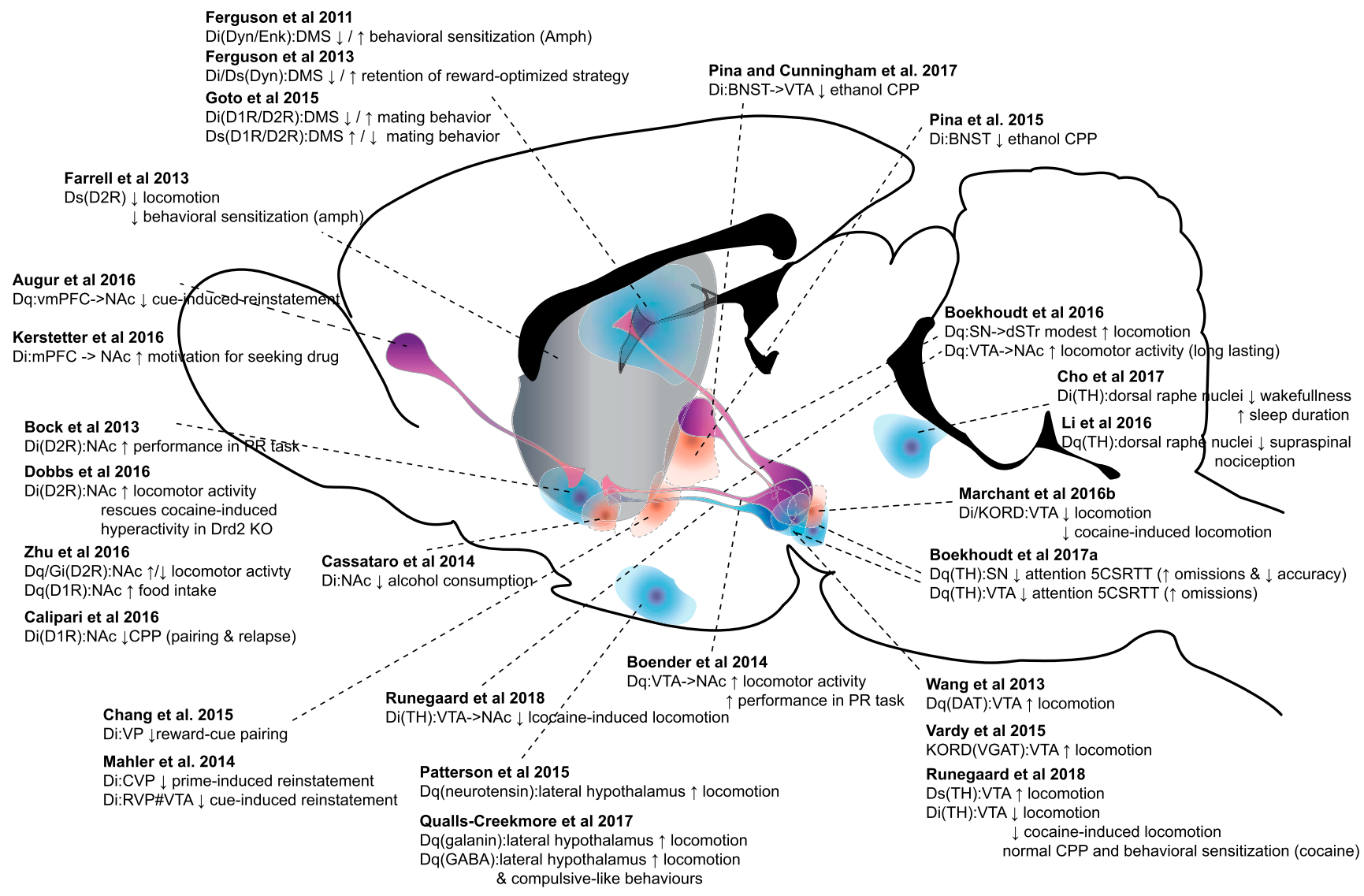

KEY: DREADD(promotor limiting expression if DIO):injectionsite ->injectionsite 2 if projectionspecific behavioral effect

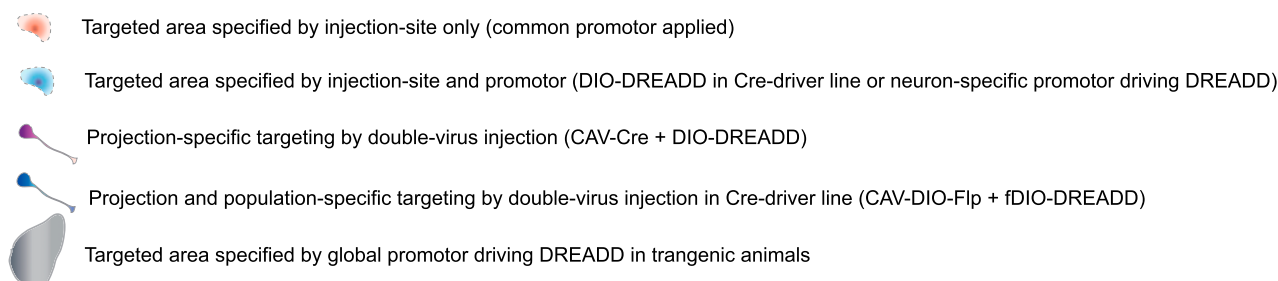

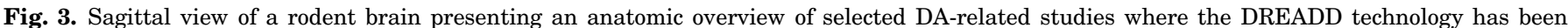

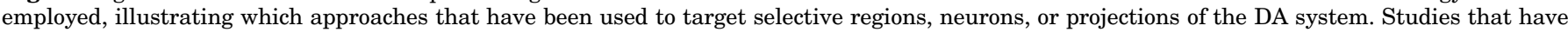

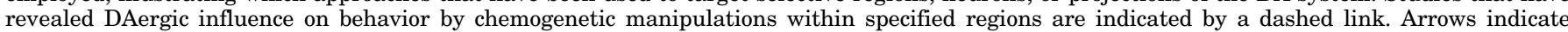

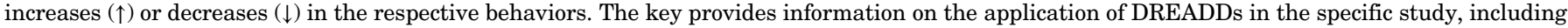

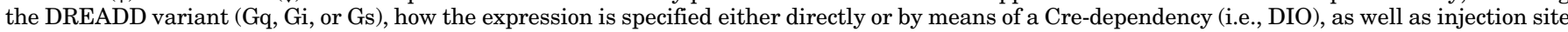

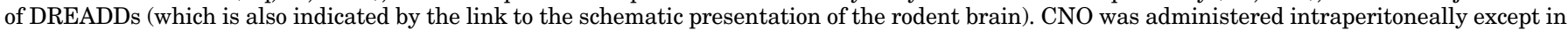

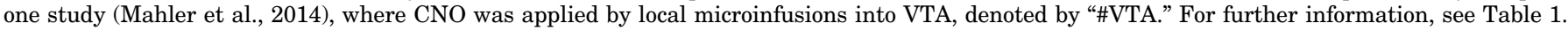




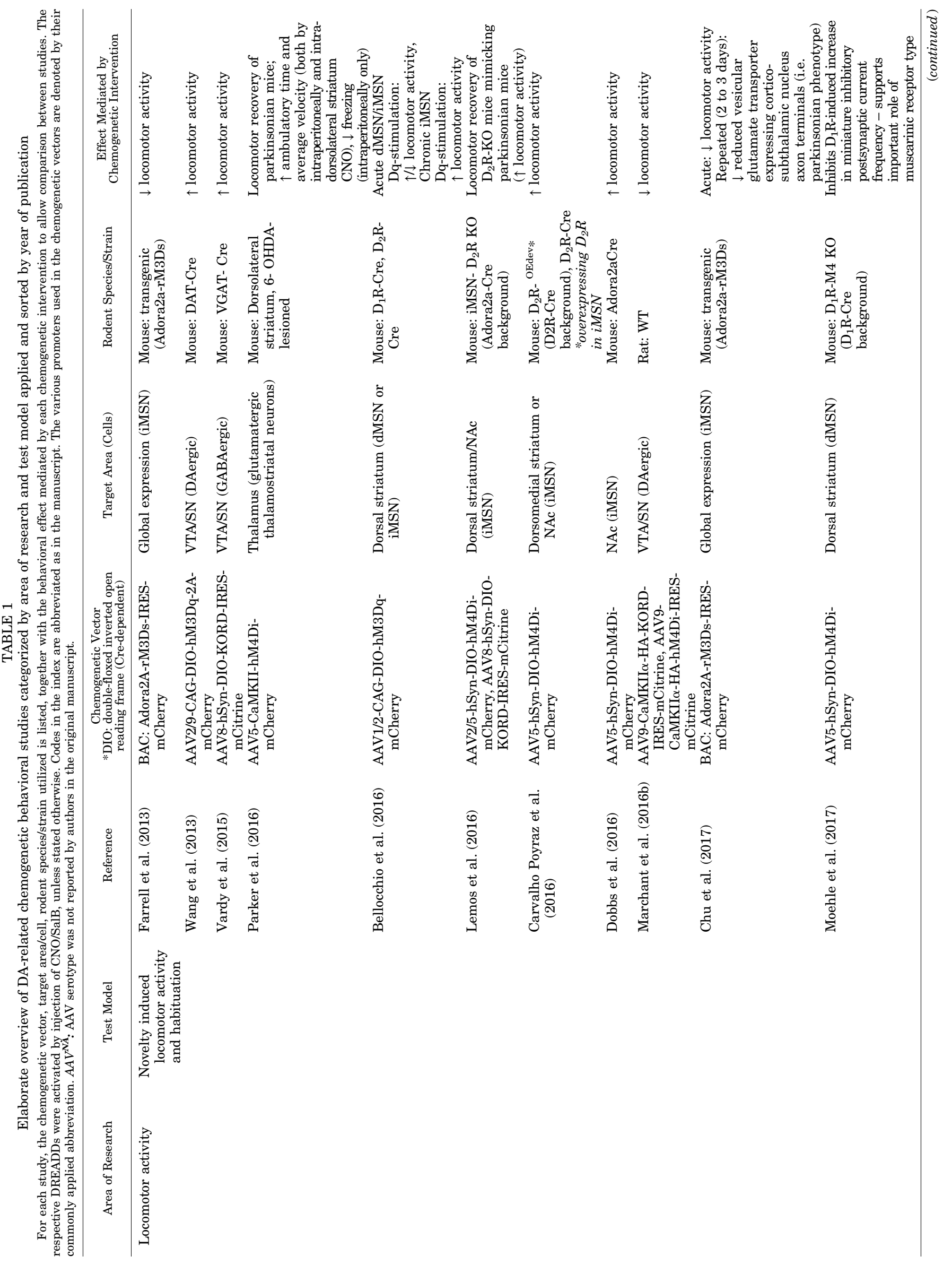




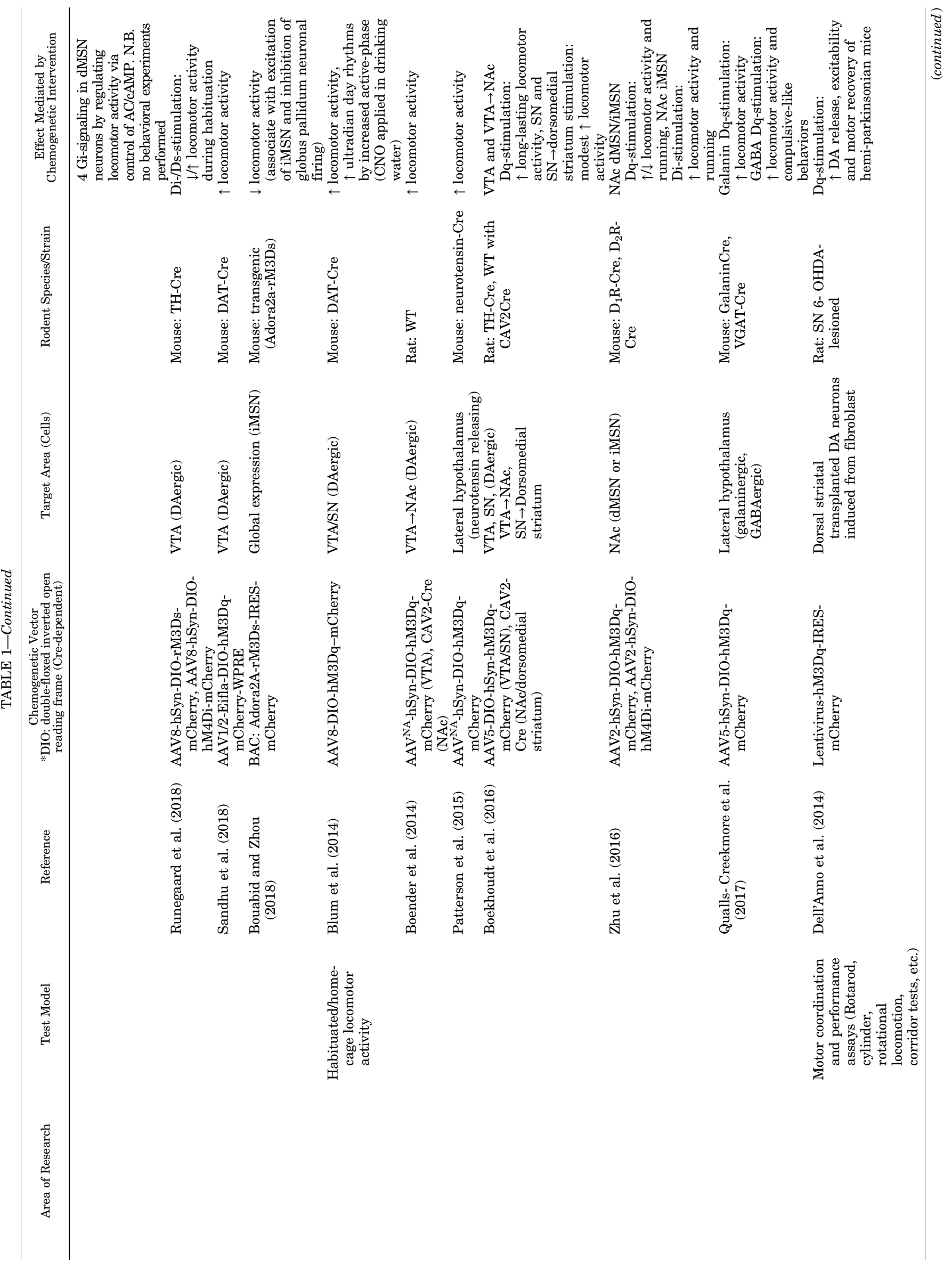




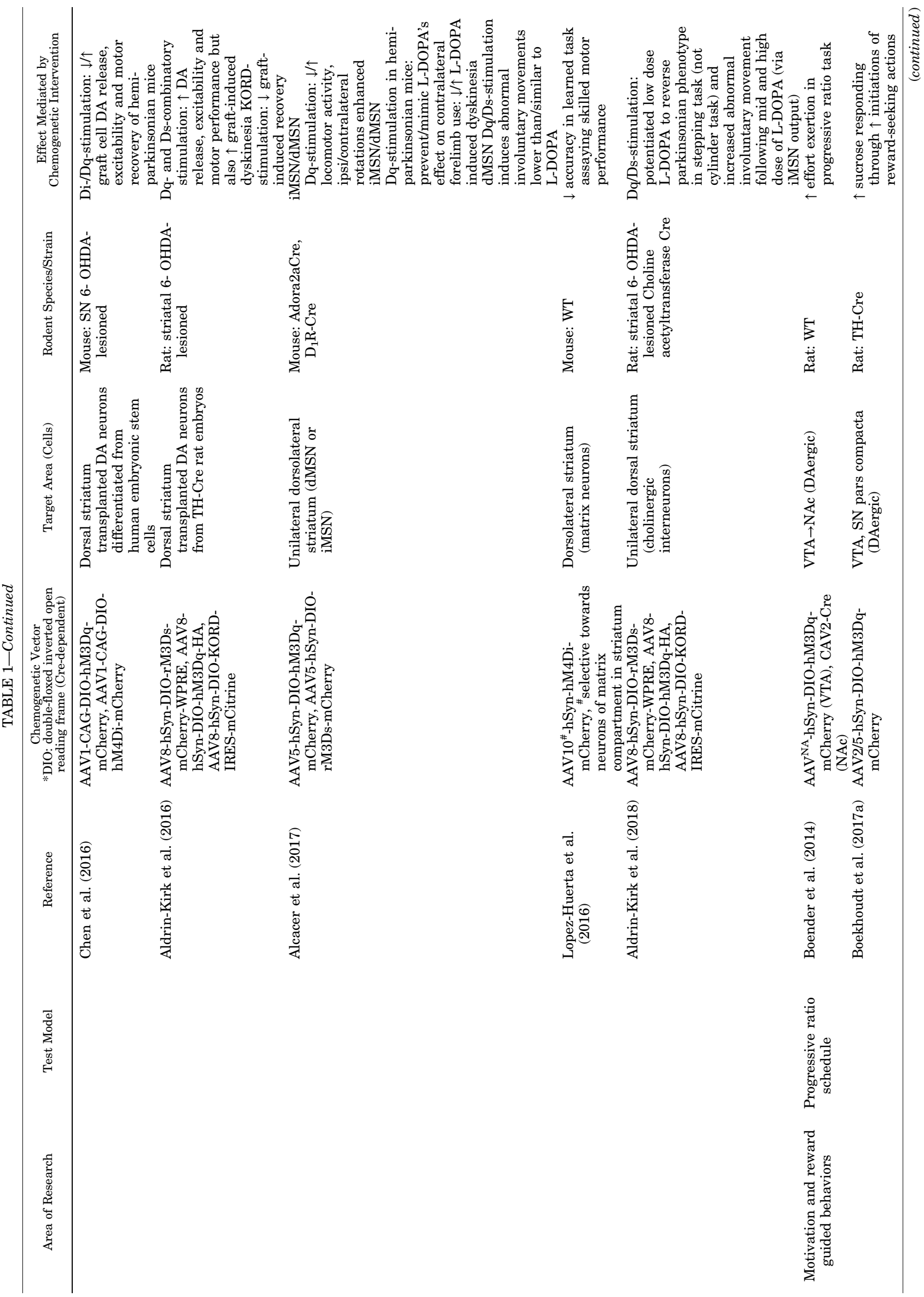




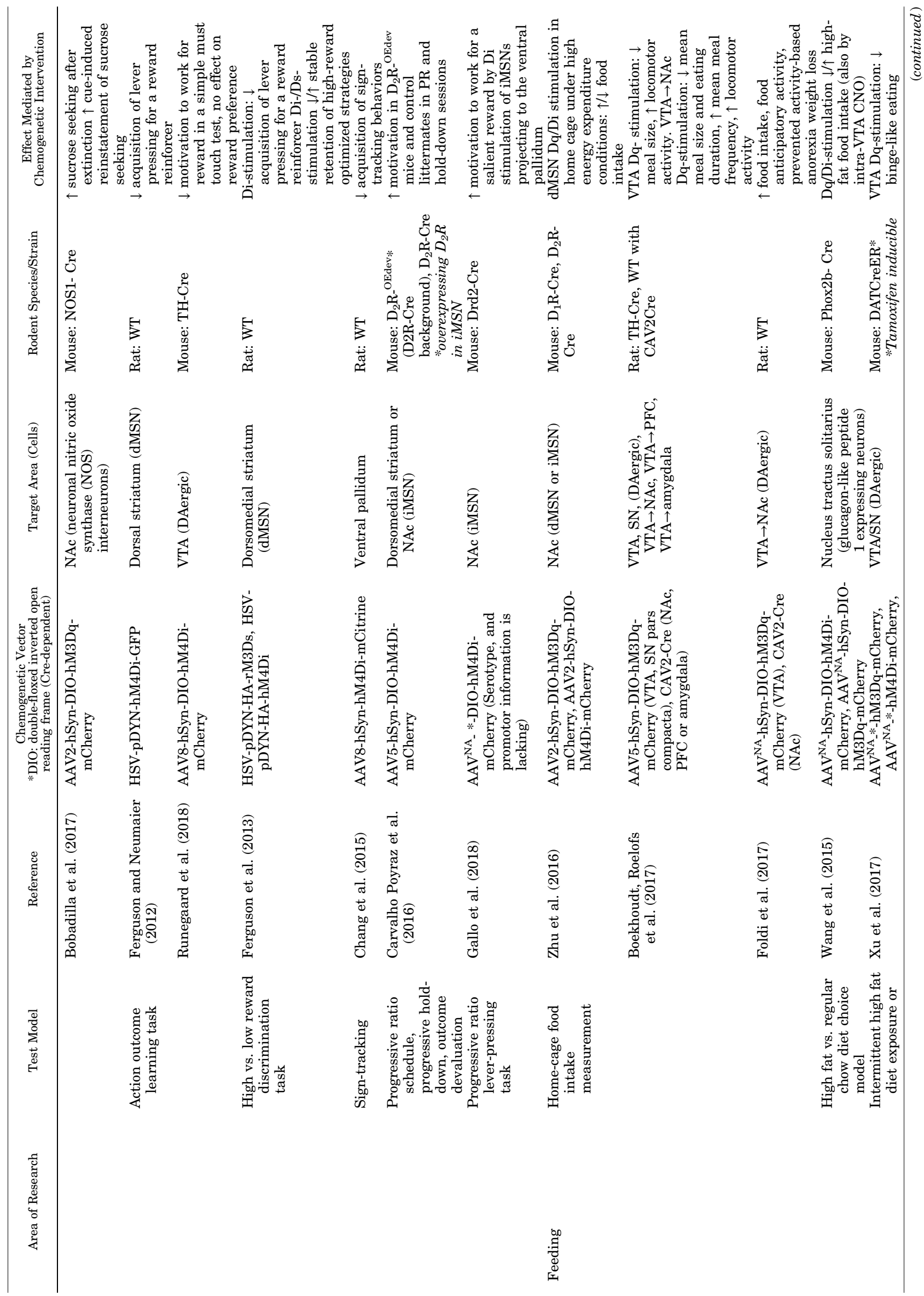




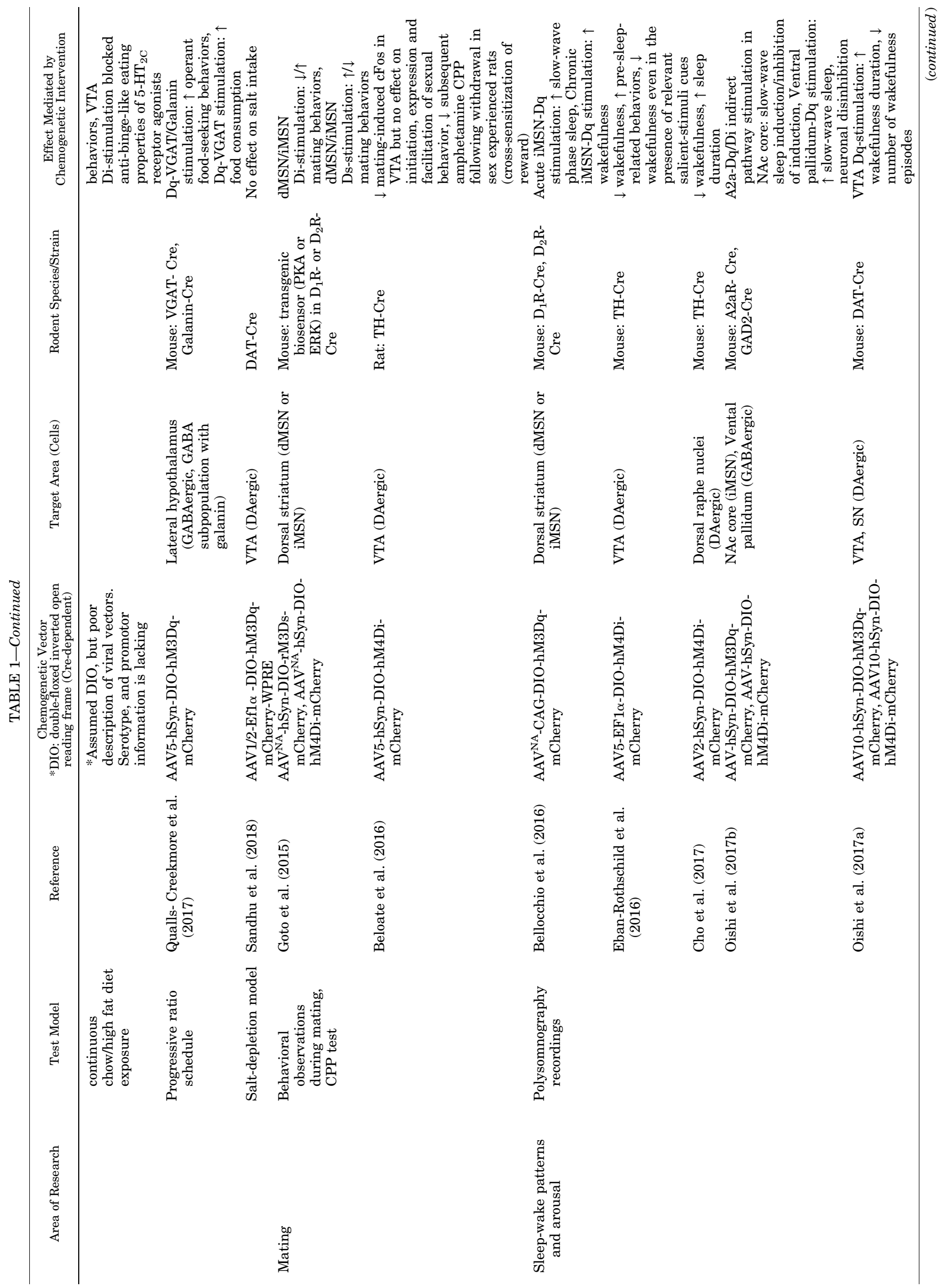




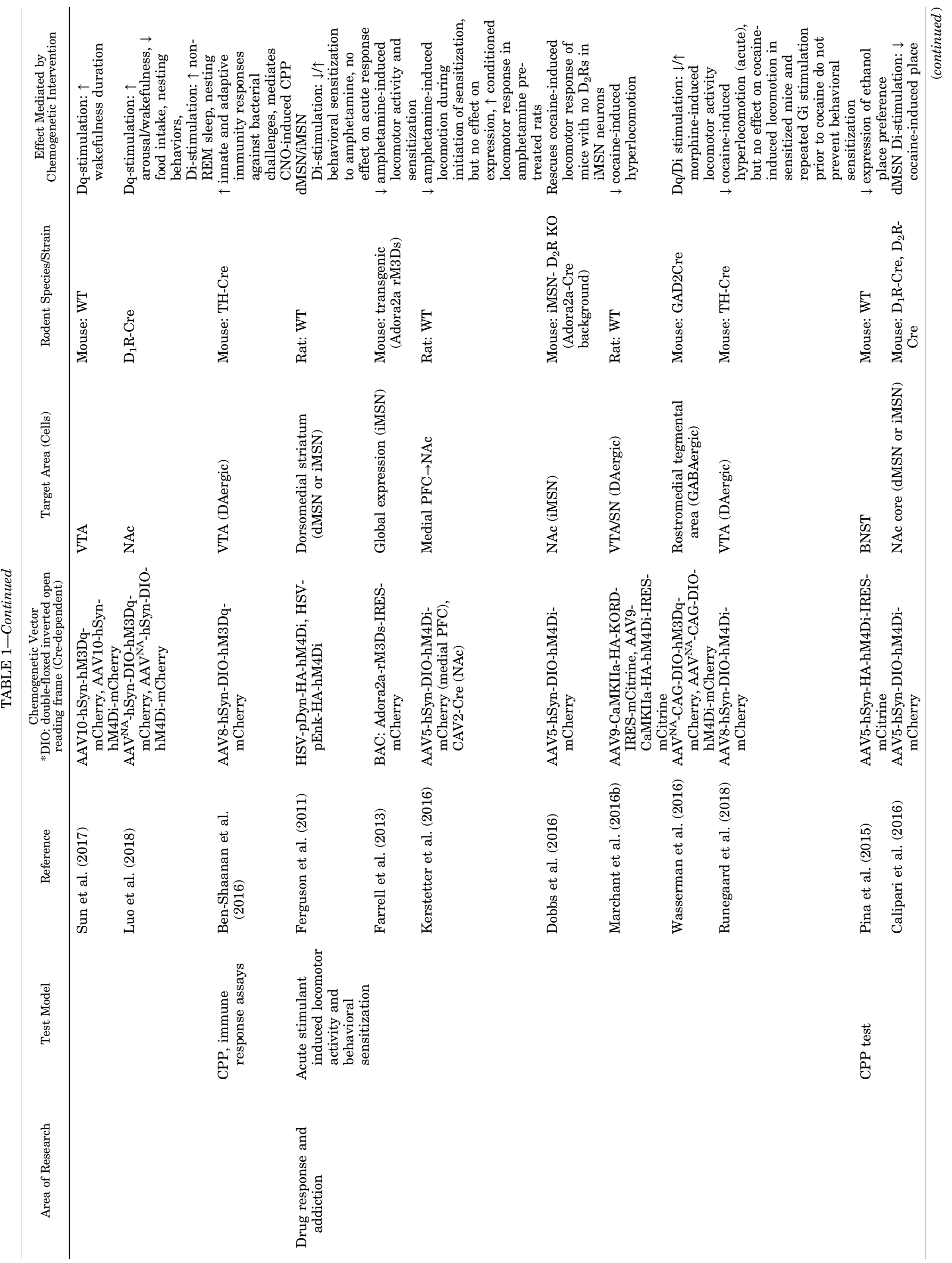




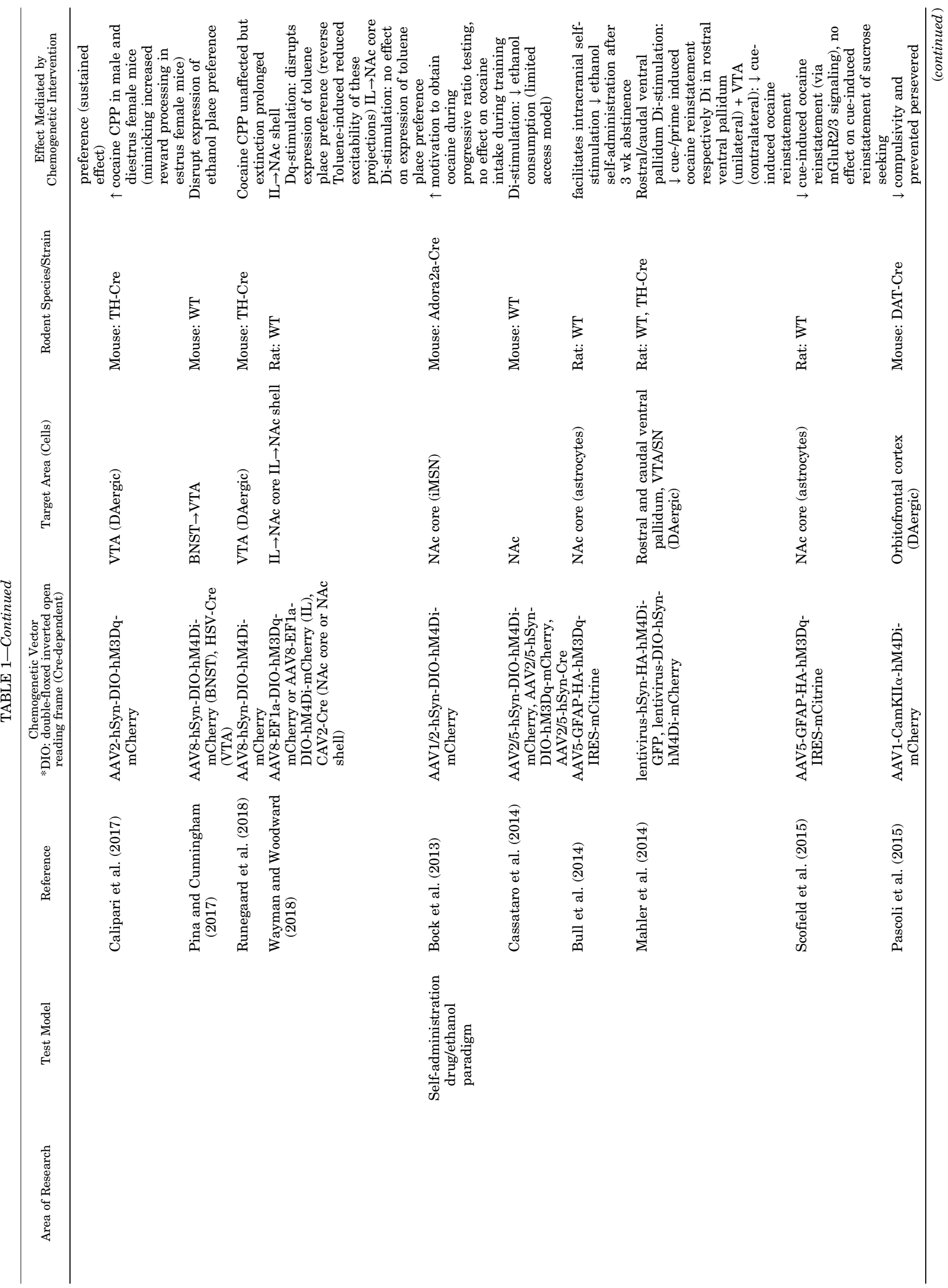




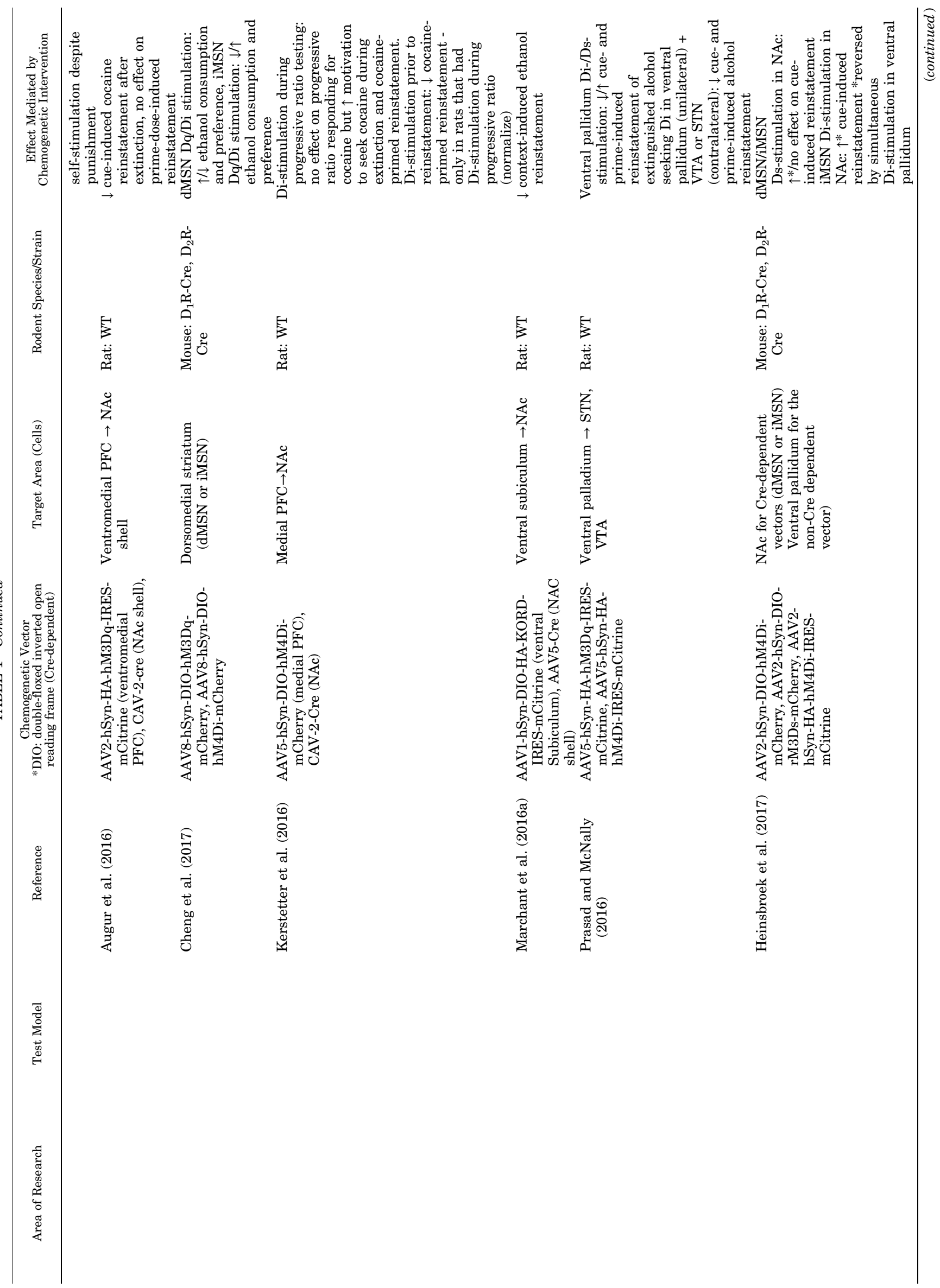




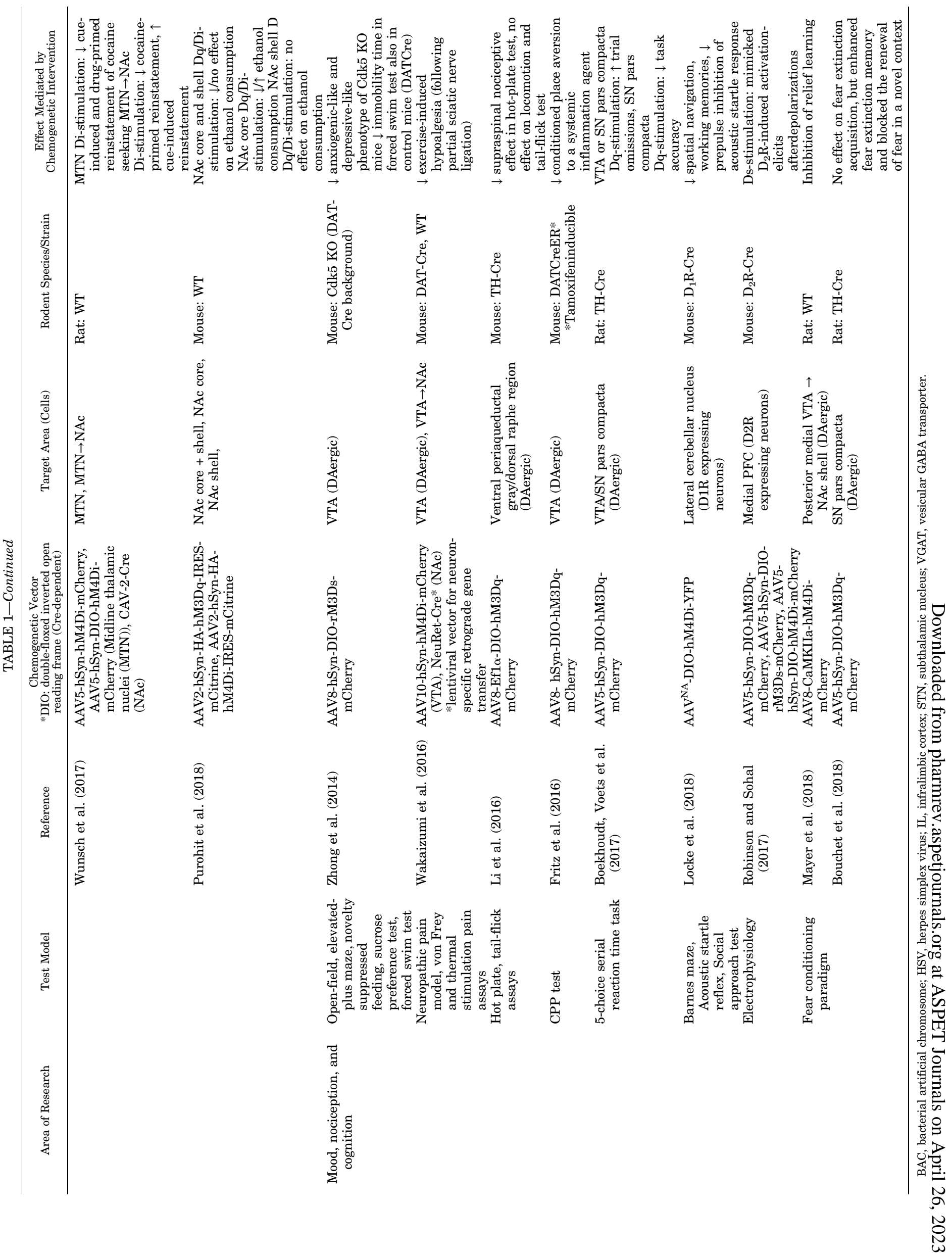


of chemogenetics (Smith et al., 2016; Whissell et al., 2016).

\section{A. Locomotor Activity}

1. Dopamine Activity Correlates with Locomotor Activity-Proof-of-Concept Studies. Classic behavioral pharmacology experiments have indicated that locomotor hyperactivity can be attributed to increased striatal DA levels (Pijnenburg et al., 1976). Therefore, initial DREADD investigations were carefully applied to behavioral neuroscience with conceptual proof-ofconcept studies in which researchers applied DREADDs to confirm pharmacologic manipulation of general neuronal populations with immediate effects on behaviors such as locomotor activity. A direct relationship between the brain DA system and regulation of locomotor activity in rodents was supported by expression of hM3Dq within midbrain (VTA/SN) DAergic neurons of DAT-Cre mice (Wang et al., 2013). CNO application increased the firing frequency of DA neurons in midbrain slices, and this finding was paralleled by strong dose-dependent hyperactivity in mice challenged with CNO (Wang et al., 2013). Likewise, in DAT-Cre mice also with hM3Dq expressed in DA midbrain neurons, the active phase of locomotor day rhythms was extended following CNO administration. This result resembled the same hyperdopaminergic phenotype that has been observed following amphetamine administration or modeled in the DAT-knockout (KO) mouse (Blum et al., 2014). With the use of a dual viral strategy to allow better spatial resolution of specific DA projections, analogous results were demonstrated in rats (Boender et al., 2014). Here, projection-specific expression of hM3Dq in VTA-NAc neurons was obtained by combined use of the retrogradely transported CAV2-Cre virus injected into the NAc and an AAV-encoding Credependent DREADD vector injected into the VTA of WT rats. This study also showed long-lasting increases in locomotor activity $(3-7 \mathrm{~h})$. These findings were subsequently substantiated by selective expression of viral hM3Dq in TH-Cre rats, and engagement of TH-positive (i.e., considered DAergic) neurons of the VTA, but not the SN pars compacta, demonstrated similar longlasting hyperlocomotion (Boekhoudt et al., 2016). This is consistent with older pharmacological data and literature supporting a key role of VTA DA neurons in the regulation of hyperactivity, while SN DA neurons appear to be more involved in other aspects of locomotion such as motor coordination (Delfs et al., 1990; Barter et al., 2014). We also recently demonstrated clear bidirectional regulation of locomotor activity during habituation to a novel environment in TH-Cre mice injected in VTA with Cre-dependent rM3Ds and hM4Di (Runegaard et al., 2018). In this study, CNO pretreatment increased and reduced locomotor activity in rM3Ds- and hM4Di-expressing mice, respectively, while CNO had no effects in WT animals (Fig. 4).
Exploratory locomotor activity has also been shown to be reduced by SalB or CNO in WT rats expressing KORD or hM4Di, respectively, in the midbrain (Marchant et al., 2016b). Locomotor activity can be indirectly manipulated by expressing KORD in midbrain GABAergic interneurons, which upon SalB administration resulted in disinhibition of VTA DA neurons and increased locomotor activity (Vardy et al., 2015). Summarized, these studies substantiate already compelling evidence that DA signaling directly regulates locomotor activity and confirms well-established pharmacological evidence that the mesolimbic pathway, comprising VTA to NAc projecting DA neurons, enforces this effect (Delfs et al., 1990; dela Peña et al., 2015). Also, the studies support local circuits within the VTA that act as central regulatory components of locomotor activity.

2. The Relative Output-Activity from Direct Medium Spiny Neurons and Indirect Medium Spiny Neurons Determines Locomotor Activity. A simple direct relationship between DA and locomotor activity, as described above, is, however, challenged by the hypofunctional DA system seen in ADHD patients, although this has been suggested to be explained by alterations in postsynaptic DA receptor signaling (Volkow et al., 2009; Napolitano et al., 2010). Accordingly, many chemogenetic studies have focused on mimicking and/or manipulating DAergic receptor signaling directly in striatal MSN subpopulations, encompassing Gs-coupled $\mathrm{D}_{1} \mathrm{R}$ expressing striatonigral neurons (dMSNs), belonging to the direct pathway, and Gi-coupled $\mathrm{D}_{2} \mathrm{R}$ expressing striatopallidal neurons (iMSNs), belonging to the indirect pathway. In a transgenic mouse expressing Gs-coupled rM3Ds throughout all iMSNs, exploratory locomotor activity was strongly reduced upon CNO administration (Farrell et al., 2013; Chu et al., 2017; Bouabid and Zhou, 2018). This was recently linked to inhibition of globus pallidus neuronal firing as a consequence of rM3Ds-mediated increased excitation of the iMSNs (Bouabid and Zhou, 2018). This suggests that AC/cAMP signaling in MSNs plays a significant role in regulating locomotor activity, which is in line with a chemogenetic study demonstrating an important role of Gi-coupled signaling in dMSNs in regulating locomotion (Moehle et al., 2017). It was correspondingly shown that hM4Di stimulation in iMSN of anatomically defined striatal areas, including the dorsomedial striatum and NAc, increased locomotor activity (Carvalho Poyraz et al., 2016). Moreover, acute CNO administration led to increased and decreased locomotor activity in mice with dorsostriatal expression of $h M 3 D q$ in dMSNs of $D_{1} R$-Cre mice and in iMSNs of $D_{2} R$-Cre or A2a-Cre mice, respectively (Bellocchio et al., 2016; Alcacer et al., 2017). Interestingly, dissection of accumbal efferents revealed a stronger influence of the indirect pathway on locomotor activity. While significant bidirectional control of basal locomotor activity was 
A

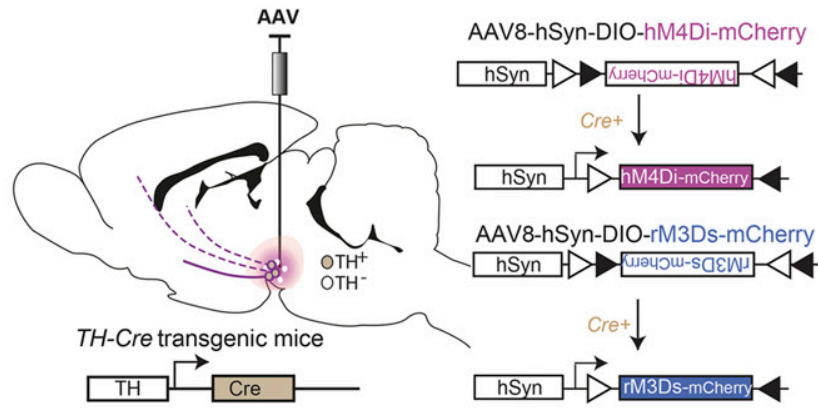

C

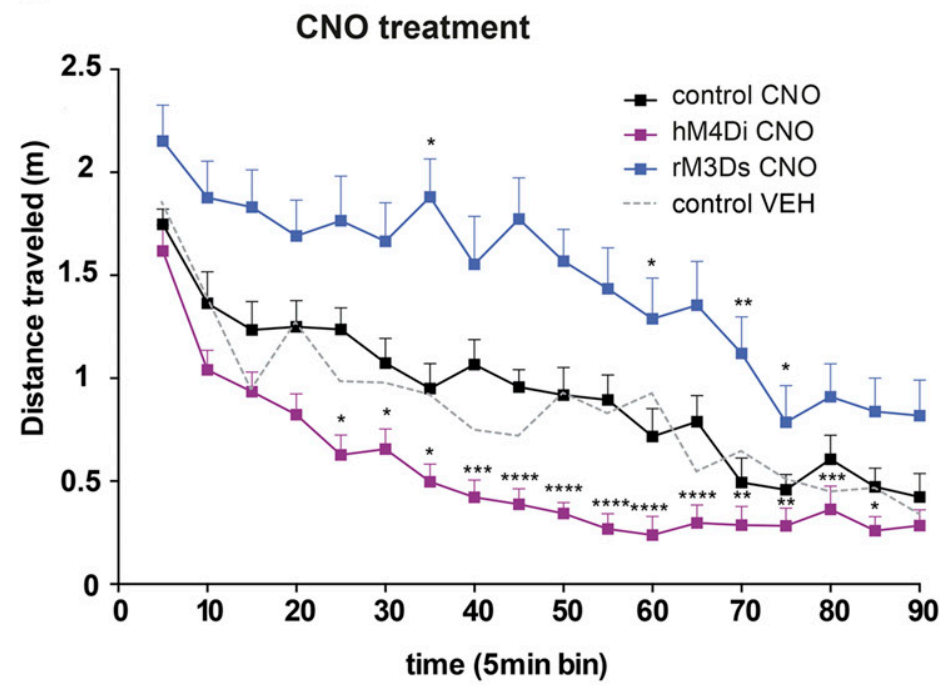

B

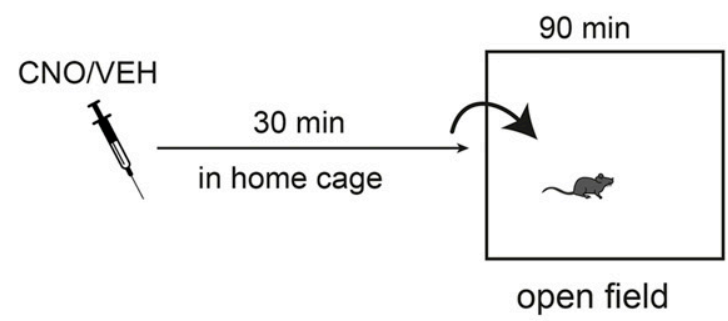

D

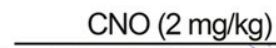

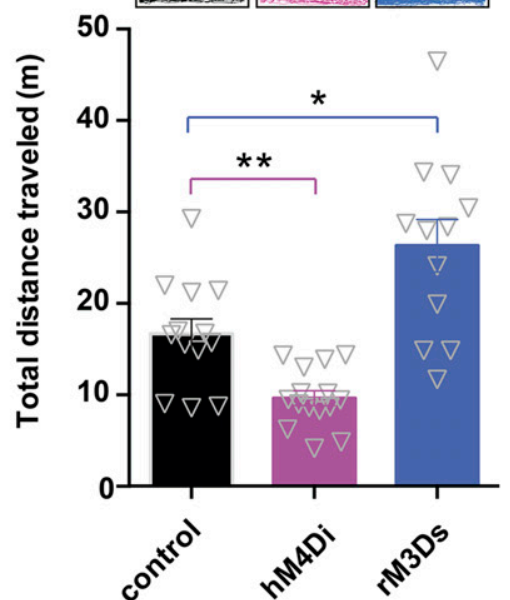

Fig. 4. Bidirectional effects on locomotor habituation by hM4Di and rM3Ds stimulation in VTA [modified and adapted from Runegaard et al. (2018)]. (A) AAV carrying hM4Di or rM3Ds was injected into the VTA of TH-Cre or WT mice. (B) Experimental timeline to assess habituation of noveltyinduced locomotor activity. CNO was injected intraperitoneally in the home cage 30 min prior to placement into the center of an open field where locomotor activity was recorded and tracked for $90 \mathrm{~min}$. (C) Time course of the 90-min habituation of novelty-induced locomotor activity in an open field 30 min after CNO injections in control (black), hM4Di-(magenta), or rM3Ds-(blue) expressing mice. Control mice injected with vehicle were included and compared with CNO-treated control mice to assess the effect of CNO alone, as shown by a dashed gray line. CNO demonstrated bidirectional control of novelty-induced exploratory activity during habituation in hM4Di and rM3Ds mice while leaving WT mice unaffected $(* * * * P<0.0001$; *** $P<0.001$; $* * P<0.01 ; * P<0.05$ relative to control). (D) Representative tracks and graphs of total distance traveled during the habituation. Analysis of the total distance traveled showed significant reduction and increase in novelty-induced exploration following CNO in hM4Di and rM3Ds mice, respectively, compared with WT controls $\left({ }^{*} P<0.05 ; * P<0.01\right.$ relative to CNO control, $\mathrm{N}: 15 \mathrm{hM} 4 \mathrm{Di} \mathrm{CNO}, 12 \mathrm{rM} 3 \mathrm{Ds} \mathrm{CNO}, 13$ control CNO). Data are shown as means + S.E.M.

demonstrated by NAc iMSNs with reduced and increased activity following hM3Dq or hM4Di stimulation, respectively, only slight increases in locomotion were observed with hM3Dq activation of dMSNs in NAc (Zhu et al., 2016). These observations collectively support a major influence of $\mathrm{D}_{2} \mathrm{R}$ activation in iMSNs on the activity of dMSNs and thereby on locomotor activity. Notably, this is consistent with a new theory of lateral inhibition of iMSNs onto dMSNs (Dobbs et al., 2016) and also with evidence from rodent $\mathrm{KO}$ studies, indicating that $M S N D_{1} R$ and $D_{2} R$ interactions control locomotor activity in a synergistic, balanced manner (Welter et al., 2007). For a comprehensive review of DA receptor KO mouse behaviors, see Holmes et al. (2004).

3. Locomotor Activity-A Simple Behavior with Multiple Levels of Regulation. Midbrain DA neurons receive and integrate inputs from multiple brain regions, as well as from local interneurons. DREADD-induced manipulations of different midbrain DA-projection neurons confirm the importance of these neurons for regulating DA activity, release, and behaviors including locomotion. As mentioned above, chemogenetic inhibition by KORD in VTA GABAergic interneurons increased locomotor activity as a consequence of VTA DA disinhibition (Vardy et al., 2015). GABAergic neurons residing within the lateral hypothalamus were recently found to alter locomotor activity via direct and indirect regulation of the VTA, i.e., hM3Dq stimulation of both VTA-projecting (i.e., GABA-expressing) neurons and non-VTA-projecting (i.e., galanin-expressing) neurons increased locomotor activity (Qualls-Creekmore et al., 2017). Similarly, CNO-induced release of neurotensin in the mouse VTA, with hM3Dq expression confined to lateral hypothalamic neurons expressing neurotensin, induced prolonged DA-dependent locomotor activity and DA release in the NAc via neurotensin receptor 
1 expressed on VTA DA neurons (Patterson et al., 2015). Furthermore, morphine-induced hyperlocomotion was regulated by DREADD-mediated modulation of rostromedial tegmental nucleus GABA interneurons targeting NAcprojecting DA neurons (Wasserman et al., 2016). While there was no effect of either DREADDs on basal locomotor activity, hM4Di and hM3Dq prevented morphine-induced hyperlocomotion, possibly by changing the firing thresholds for GABA interneurons and thereby altering their inhibitory effect on NAc-projecting DA neurons (Wasserman et al., 2016). Note that chemogenetic interventions of locomotor response to other drugs such as cocaine and amphetamine are addressed in section II.C.1.

4. Dysfunctional Motor Control in Parkinson's Disease. In contrast to locomotor activity, which appears to be regulated predominantly by midbrain DA neurons projecting to ventral parts of the striatum, motor control and initiation of movement are more dependent on dorsal striatal DA neurotransmission. This is particularly evident in PD, where major symptoms include bradykinesia, akinesia, tremors, and movement rigidity (Vazey and Aston-Jones, 2013). These motor disabilities are associated with major loss of DAergic neurons within the SN, resulting in progressive loss of dorsostriatal DA input. A classic and commonly used rodent/primate model of PD is unilateral 6-hydroxydopamine (6-OHDA)-induced lesion of striatal terminal neurons that is believed to recapitulate key changes in basal ganglia circuitry and pharmacology observed in patients with PD (Ungerstedt and Arbuthnott, 1970). The resulting hemi-parkinsonian rodents exhibit severe behavioral imbalances that can be restored by L-3, 4-dihydroxyphenylalanine (L-DOPA), the DA precursor applied for therapeutic use in PD patients. In nonlesioned control mice, unilateral expression and stimulation of hM3Dq in dMSNs or iMSNs of the dorsal striatum induced contra- and ipsilateral rotations, respectively (Alcacer et al., 2017). In lesioned hemi-parkinsonian mice, unilateral stimulation of dMSN in the DA-denervated striatum mimicked the therapeutic effect of L-DOPA on contralateral forelimb use, as assessed in a cylinder test, while stimulation of iMSN prevented L-DOPA's effect in these mice (Alcacer et al., 2017). The findings are noteworthy, and perhaps relevant for future clinical intervention, as prolonged L-DOPA treatment is associated with dyskinesia, a debilitating and treatment-limiting condition affecting up to $80 \%$ of PD patients within 10 years (Manson et al., 2012). In the Alcacer et al. (2017) study, it was also shown that L-DOPA-induced dyskinesia or involuntary movements in hemi-parkinsonian mice were reduced by hM3Dq expressed in iMSNs on the denervated striatal site. However, stimulation of rM3Ds or hM3Dq in dMSNs of the denervated striatal site induced abnormal involuntary movements, with the most severe effect seen upon rM3Ds stimulation that resulted in L-DOPA-like induced dyskinesia (Alcacer et al., 2017). This result fully complements recent findings using a $\mathrm{C}$-fos driver mouse line and optogenetics to probe the origin of L-DOPA-induced dyskinesia (Girasole et al., 2018).

Fetal DA neuron transplantation trials in PD patients suggest that motor disturbances can be normalized in some patients where L-DOPA treatment is inefficient or associated with dyskinesia. The efficiency of the neuronal transplantation, however, is highly variable (Barker et al., 2013). Chemogenetics were recently employed in rodent models to investigate whether the therapeutic efficacy can be improved by activating the excitatory hM3Dq within engrafted neurons. Strikingly, such manipulations did lead to increased excitability and DA release within induced or neuroblastic DA neurons transplanted into the dorsal striatum of 6-OHDA-lesioned mice. Moreover, the manipulations rescued motor deficits in mice (Chen et al., 2016) and rats (Dell'Anno et al., 2014; Aldrin-Kirk et al., 2016). Aldrin-Kirk et al. (2016) even went on to simultaneously express and activate hM3Dq and rM3Ds in the grafted cells. With this, they induced dyskinesia similar to what develops as a side-effect in some patients following transplantation of DAergic neurons. They suggested a mechanism for graft-induced dyskinesia that involves $5-\mathrm{HT}_{6}$ receptors causing dysplastic DA release from transplants by activating Gs-signaling that in turn counteracts autoinhibitory control by $\mathrm{D}_{2} \mathrm{Rs}$ (Aldrin-Kirk et al., 2016).

A recent chemogenetic study adds further support to the hypothesis that parkinsonian-related motor deficits are caused by maladaptive, relative regulation of the direct and indirect pathways of basal ganglia circuitry, rather than by reduced absolute DA levels (Parker et al., 2016). The severity of motor deficits observed in 6-OHDA-lesioned mice was correlated with a reversed relative strength of excitatory thalamic drive onto direct and indirect pathway MSNs with a bias toward the indirect pathway. Inhibition of this thalamostriatal input, which drives maladaptive asymmetric activation of basal ganglia by activating hM4Di in thalamostriatal terminals, restored motor function in DA-depleted mice (Parker et al., 2016). Likewise, in 6-OHDA-lesioned rats, stimulation of cholinergic interneurons in the striatum via $\mathrm{hM} 3 \mathrm{Dq} / \mathrm{rM} 3 \mathrm{Ds}$ potentiated the therapeutic effects of low-dose L-DOPA (i.e., $1 \mathrm{mg} / \mathrm{kg}$ ) and facilitated L-DOPA-induced abnormal involuntary movement after moderate and high doses (i.e., 3 and $9 \mathrm{mg} / \mathrm{kg}$ ) of L-DOPA—effects that appeared to be driven primarily through the iMSN pathway since the same effect was induced by the D2 agonist quinpirole (Aldrin-Kirk et al., 2018). Further support for maladaptive regulation between the direct and indirect pathways was achieved in a study in which bradykinesia was modeled in a genetic mouse model with selective deletion of the $D_{2} R$ in iMSNs (Lemos et al., 2016). The degree of 
bradykinesia correlated with the enhanced GABAergic transmission downstream of iMSNs, and GABAergic tone and locomotor control were restored through stimulation of Gi-signaling in iMSNs by activating hM4Di expressed in iMSNs of the dorsal striatum and NAc to mimic $\mathrm{D}_{2} \mathrm{R}$ function (Lemos et al., 2016). Lastly, a chemogenetic approach has been applied to investigate the role of striatal striosome and matrix compartments in procedural learning and use of motor skill. The study supported that there is no synaptic connectivity between these two compartments. Nevertheless, by selective transduction of matrix neurons only with hM4Di, evidence was obtained for a role of dorsolateral striatal matrix neurons in forepaw use (Lopez-Huerta et al., 2016).

\section{B. Motivation and Reward-Guided Behaviors}

DAergic-mediated regulation of motivation and reward-guided behaviors has garnered much attention not least due to its importance in pathologic states such as addiction and obesity (Baik, 2013). The below mentioned DREADD studies have assessed DA behavioral regulation in rodent test models in both homeostatic and maladaptive reward-related states that take advantage of an organism's desire to survive (Salamone et al., 2012). As such, operant conditioning and learning tasks involve reward reinforcement and punishment processes that lead to behavioral acquisition, which regulates the probability, proximity and availability of relevant stimuli in rodent tasks such as progressive ratio scheduling (Richardson and Roberts, 1996), operant learning (Todd et al., 2014), and cue/sign-tracking (Salamone et al., 2012).

1. Substantiating Dopamine as a Key Regulator of Motivation. Motivation deficits represent a key feature of psychiatric disorders with DA being a critical neural substrate (Baik, 2013). Through the use of progressive ratio scheduling to assess motivational behaviors, Boender et al. (2014) demonstrated that hM3Dq-mediated activation of the mesolimbic DAergic pathway, as targeted through use of a Cre-recombinase expressing CAV2 vector, increased progressive ratio performance, and hence effort exertion. The same research group refined their methodological approach to show that this enhanced motivation was specifically due to increased action response initiation and not due to other forms of motivational behaviors (Boekhoudt et al., 2018).

2. The Role of Direct Medium Spiny Neurons and Indirect Medium Spiny Neurons in Regulating RewardMotivated Behaviors. Given its importance in mediating DAergic neurotransmission, the dorsal striatum is known to play a critical role in reward-guided decision making, but the precise circuits involved have not been fully elucidated. In one study by Ferguson and Neumaier (2012), it was observed that transient hM4Di activation of dMSNs within the rat dorsal striatum impaired acquisition of an operant learning task assessing reward reinforcement learning, as supported by significantly lower lever pressing rates for a reward. In another study, the same group further investigated the role of the dorsomedial striatum in action-outcome learning using a high-versus-low reward discrimination task (Ferguson et al., 2013). Rats learned to associate lever presses with one daily alternating lever for a high-reward outcome and the other for a low-reward outcome. Upon chemogenetic modulation of dMSN signaling via activation of hM4Di or rM3Ds during training sessions, no effect was seen on task acquisition or subsequent performance, i.e., $24 \mathrm{~h}$ after the last training session. However, hM4Di activation during training sessions significantly impaired stable retention of task information, as these rats demonstrated a reduced preference for the high-reward lever and needed more trials to reach criterion, that being the choice of the high-reward lever $75 \%$ of all trials, 1 week after the last training session. Activation of rM3Ds in dMSNs produced the opposite effects and improved stable retention of this decision-making strategy 1-week posttraining. These findings provided strong evidence that activation of dMSNs in the dorsomedial striatum is essential for retaining test-specific information regarding reward-guided behaviors. Moreover, the fact that these chemogenetic manipulations of dMSNs affected behaviors 1 week post-CNO administration suggested that significant neuronal plasticity changes took place within these neurons during acquisition of this task (Ferguson et al., 2013).

DREADD approaches, together with an operant lever-pressing task with progressive ratio schedules, were used to assess the influence of iMSNs in NAc and dorsomedial striatum on motivational processes. Activation of hM4Di expressed in iMSNs of either the dorsomedial striatum or NAc reversed motivational deficits in mice genetically manipulated to overexpress $\mathrm{D}_{2} \mathrm{Rs}$ and also in WT controls. These effects were due to enhanced response initiation without goal-guided efficiency, motivational vigor, or sensitivities relating to upor down-valuation of reward being affected (Carvalho Poyraz et al., 2016). Hence, Gi-signaling in iMSNs appeared to increase motivation to work for reward, probably as a result of reduced GABAergic tone in target areas or directly onto neighboring dMSNs causing disinhibition (Dobbs et al., 2016). This is further supported by a study by Gallo et al substantiating a role of the ventral pallidum. It was shown in this study that activation of hM4Di expressed in NAc iMSNs projecting to the ventral pallidum increased motivation to work for a salient reward in a lever-pressing task with progressive ratio schedule (Gallo et al., 2018). Of additional interest, selective hM3Dq-stimulation of NAc interneurons expressing nitric oxide synthase was demonstrated to increase sucrose seeking after extinction and cue-induced reinstatement (Bobadilla et al., 2017). 
Together, these studies suggest that DA-dependent, reward-motivated behaviors are regulated by multiple pathways that depend on the output of both dorsal striatal and NAc MSNs. Importantly, while the chemogenetic manipulations of MSN activity described above tend to be considered a direct reflection of DA transmission with the Gi- and Gs-coupled DREADDs directly modulating DA receptor signaling, it should be noted that these neurons express multiple different GPCRs that signal through similar cascades. Accordingly, the behaviors studied are not regulated solely by DA transmission but also by other transmitter systems acting through different sets of GPCRs.

3. Feeding. Food activates neural circuits associated with reward and DAergic signaling, particularly in the NAc, midbrain, and amygdala. It is widely believed that the DA mesolimbic pathway facilitates associative learning between food-related rewards and feedingrelated environments, with food or food-related cues initiating phasic mesolimbic DAergic firing and behaviors associated with acquiring food (Baik, 2013). Boekhoudt et al. (2017a) investigated whether chemogenetic activation of midbrain DAergic neurons promoted or reduced feeding to establish a causal relationship between DAergic activity and food intake. They measured home-cage feeding patterns while concurrently stimulating hM3Dq in the SN pars compacta or selective VTA subpopulation projections toward the NAc, amygdala, or PFC. They found that general stimulation of hM3Dq in VTA reduced meal size, while at the same time increasing locomotor activity. Specific mesolimbic pathway DA activation disrupted feeding patterns by reducing mean meal size and duration but increased the frequency of meal initiations (Boekhoudt et al., 2017a). Although, the mesolimbic DA pathway seemed to both promote and reduce food intake in that study by facilitating feeding initiation and cessation behaviors (Boekhoudt et al., 2017a), chemogenetic stimulation in the same mesolimbic pathway in another study prevented anorexic weight loss in an activity-based rat model of anorexia (Foldi et al., 2017). When rats were given time-limited daily access to food, hM3Dq stimulation of VTA DAergic neurons projecting to the NAc increased food intake and food anticipatory activity and blocked anhedonia-associated weight loss and subsequent death (Foldi et al., 2017).

Specific chemogenetic activation of dMSNs or iMSNs within the NAc was done to assess food intake under both low and high-energy expenditure (i.e., a running wheel was placed in the home cage) conditions. The data showed that hM3Dq and hM4Di activation of NAc dMSNs enhanced and diminished food intake, respectively, in high-expenditure conditions when the running wheel was placed in the animals' home cages for 14 days. Interestingly, the same study found that NAc dMSN hM3Dq-stimulation reduced overall food intake in low expenditure conditions (i.e., open field test) over the course of $24 \mathrm{~h}$ (Zhu et al., 2016). These studies support and complement the hypothesis that DAergic pathways, particularly the mesolimbic, regulate homeostatic energy expenditure and feeding patterns. In addition, altered DAergic signaling patterns, induced by chemogenetic manipulations, were consistently found to disrupt feeding patterns, indicating that tightly controlled and optimal DA neurotransmission exists to regulate feeding.

As outlined below, there is strong evidence suggesting that serotonin directly acts on VTA DA neurons to reduce food intake, linking the two transmitters together in regulation of feeding behaviors. As well as finding that stimulation of hM3Dq expressed in VTA DA neurons inhibited binge-like eating in mice on a high-fat diet, $\mathrm{Xu}$ et al. (2017) demonstrated that the $5-\mathrm{HT}_{2 \mathrm{C}}$ receptor agonist lorcaserin, which has been approved for obesity treatment (Martin et al., 2011), and the 5-HT-releasing agent fenfluramine inhibited these eating patterns via $5-\mathrm{HT}_{2 \mathrm{C}}$ receptor activation. This effect was directly linked to an increase in VTA DAergic signaling and further supported by the finding that VTA DA hM4Di activation prevented fenfluramine from suppressing binge-like eating patterns (Xu et al., 2017). The same group subsequently demonstrated that $\mathrm{hM} 3 \mathrm{Dq}$-mediated activation of VTA $5-\mathrm{HT}_{2 \mathrm{C}}$ receptorexpressing neurons reduced ad libitum food intake, as well as lowered progressive ratio responding for sugar pellets and incentive value for food (Valencia-Torres et al., 2017). The studies substantiate that $5-\mathrm{HT}_{2 \mathrm{C}}$ agonism inhibits food-related motivational processes at least in part via direct effects on midbrain DAergic transmission.

Application of chemogenetics has also shown that neurons within the nucleus tractus solitarius in the brain stem can regulate food intake by affecting VTA DA neuronal activity. Activation of hM3Dq in these neurons suppressed high-fat chow diet intake by diminishing signaling of VTA DAergic neuron projecting to the NAc shell (Wang et al., 2015). Notably, it was found that this likely was the result of increased release of glucagon-like-peptide 1 acting through GLP-1 receptors on DA VTA neurons, leading to reduced excitatory synaptic strength (Wang et al., 2015). Likewise, hM3Dqmediated activation of the lateral hypothalamic GABAergic neurons caused an overall increase in chow diet consumption as a consequence of direct VTA innervation (Qualls-Creekmore et al., 2017). These studies highlight how different brain regions and neurochemical systems can modulate DA-dependent signaling and behaviors and how chemogenetics can aid elucidation of previously unknown pathways.

4. Mating. During sexual behaviors, VTA $\mu$-opioid receptor-expressing neurons are activated and DA is released into NAc and striatum (Damsma et al., 1992; Bassareo and Di Chiara, 1999; Balfour et al., 2004). In rodents, sexual behavior is normally assessed by 
pairing and allowing a male to mate with a female subject. The latency and number of investigations, intromissions, and ejaculations allow for measurement of sexual motivation, performance, memory, and reward. In rats with hM4Di expression confined to TH-positive neurons in the VTA, CNO did not affect, however, such sexual behavior (Beloate et al., 2016). Moreover, although CNO prevented VTA DA neuron activation and $\mathrm{C}$-fos induction in these neurons in response to sexual behaviors, CNO had no effect on mating-induced C-fos expression in VTA projection areas, including NAc, PFC, and basolateral amygdala. CNO was nonetheless found to impair sexual experience-induced cross-sensitization of amphetamine conditioned place preference (CPP). Thus, activation of VTA DA neurons appears not to be critical for initiation or expression of sexual behavior. Rather such activation, and thereby mesolimbic DA, increases the sensitivity to addictive drugs upon loss of the natural reward via mesolimbic plasticity established during the natural reward experience. Together, the study substantiates how DAergic VTA neurons can regulate the cross-sensitization of natural (i.e., sexual experience) and drug-related rewards (Beloate et al., 2016).

In another study, the importance during sexual behavior of dorsostriatal dMSN and iMSN activity was assessed by using genetically encoded, fluorescence resonance energy transfer-based PKA and ERK sensors together with DREADDs. The sensors were expressed in dMSNs and iMSNs, and it was found that PKA and ERK activities in these neurons rapidly switched when the male mice shifted between active and inactive phases of mating reactions. To investigate the mechanism underlying this rapid change, hM4Di or rM3Ds DREADDs were expressed in striatal dMSNs and iMSNs together with the PKA biosensor. In hM4Diexpressing dMSNs or iMSNs, CNO reduced PKA activity and concomitantly caused suppression or augmentation of positive mating reactions, respectively. In rM3Ds-expressing dMSNs or iMSNs, CNO increased PKA activity concomitantly with enhancement or suppression of positive mating reactions, respectively. Hence, the dMSNs and iMSNs of the dorsal striatum appear to differentially control mating reactions in male mice by directly regulating PKA activity (Goto et al., 2015).

5. Sleep-Wake Patterns and Arousal. Activation of VTA DAergic neurons by hM3Dq was shown to increase wakefulness periods while reducing sleep-associated behaviors, such as nest building, in both stages of the light/dark cycle in a $\mathrm{D}_{2} \mathrm{R}$-dependent manner (Oishi et al., 2017a; Sun et al., 2017). Further investigations revealed that NAc $D_{1} R$ - and NAc core adenosine A2a receptorexpressing neurons also regulated these sleep-wake patterns (Oishi et al., 2017b; Luo et al., 2018). Activation of $\mathrm{D}_{1} \mathrm{R}$ expressing neurons in the NAc by hM3Dqstimulation prolonged wakefulness and concurrently reduced food intake, while stimulation of the inhibitory hM4Di suppressed arousal and induced sleep behaviors (Luo et al., 2018). Another approach, albeit with the same chemogenetic tools, demonstrated that adenosine A2a receptor-expressing indirect pathway neurons in the NAc core can regulate slow-wave sleep, as hM3Dq or hM4Di stimulation of these neurons either induced or prevented slow-wave sleep, respectively (Oishi et al., 2017b). The studies highlight the importance of midbrain DA neuronal circuits for regulating arousal, both discovering new DA-dependent neural circuits critical for the induction and maintenance of wakefulness, projecting to the lateral hypothalamus (Luo et al., 2018) and ventral pallidum (Oishi et al., 2017b), respectively.

It was furthermore observed that acute hM3Dqactivation in dorsostriatal dMSNs increased wakefulness while reducing the time spent in the slow-wave and rapid eye movement sleep stages. Acute hM3Dqactivation of iMSNs, however, increased slow-wave phase sleep while chronic activation resulted in enhanced wakefulness (Bellocchio et al., 2016). Expectedly, one's arousal state affects motivation with the above studies revealing a direct effect of DA activity on wakefulness and sleep-wake patterns. More direct evidence for DA as a substrate integrating rewardbased motivation and arousal was obtained from data indicating that DA is critical for maintaining wakefulness even in the presence of relevant salient stimuli (Eban-Rothschild et al., 2016; Cho et al., 2017).

By using fiber photometry to measure calcium activity and polysomnographic recordings to assess wakefulness, it was observed that hM4Di-mediated inhibition of DAergic neurons in the VTA and dorsal raphe nuclei decreased wakefulness and increased sleep-related behaviors and length of sleep. This decrease in arousal occurred even when stimuli, such as accessible palatable food, a female mouse, or predator odors, were present in the same or in a neighboring cage (Eban-Rothschild et al., 2016; Cho et al., 2017). The data emphasize a plausible role governed by the DAergic system in regulating the interaction between sleep-wake patterns and reward-based motivation.

6. Immunity. When VTA neurons were acutely activated via stimulation of $h M 3 D q$ receptors expressed in these neurons $24 \mathrm{~h}$ before an Escherichia coli immune challenge, enhanced innate and adaptive immune responses were exhibited 3 - and 7-days postchallenge. In addition to increased host defense capabilities, the same VTA DA neuronal activation resulted in enhanced CPP toward the conditioned chamber. These findings indicate that VTA DAergic neurons balance their control of reward-guided behaviors with immunity or, at least, that there might be a causal relationship between enhanced VTA activity and the host's immune response to a bacterial infection (Ben-Shaanan et al., 2016). 


\section{Addiction}

Drugs of abuse, including the psychostimulants cocaine and amphetamine that act directly on the DA system, induce substantial synaptic modifications that hijack the natural reward system and cause the transition to substance addiction (Baik, 2013). Increased DA levels within the NAc have been linked to hyperlocomotion as well as to an initial perception of reward and subsequent reward prediction error guiding goal-directed behaviors (Schultz, 2007). Although the elevations in accumbal DA levels are related to both psychostimulant-induced hyperactivity (acute as well as sensitized) and general reward perception (which in rodents can be assessed indirectly by CPP or self-administration paradigms), these behaviors are likely segregated because not all addictive drugs induce hyperactivity. It has been hypothesized, however, that psychostimulant-induced locomotor hyperactivity in rodents reflects the perception of reward and could be reflective of addiction vulnerability (Deminiere et al., 1989; Piazza et al., 1989). Consistent with this hypothesis, a greater acute locomotor response to psychostimulants was found to be predictive of psychostimulant self-administration (Piazza et al., 1989) and high drug intake (Piazza et al., 2000). The rewarding effect/addictive properties of drugs of abuse and their influence on subsequent drug-related behaviors, including drug-seeking and reinstatement, are commonly assessed by CPP and selfadministration paradigms. In addition to assessing relapse following withdrawal (induced by either a cue or a prime-injection of the rewarding stimuli), the selfadministration paradigm may assess compulsive drug intake (continued self-administration despite negative consequences, such as foot shock), which is considered a major hallmark of addiction. Of further interest, we recently published a single exposure place preference protocol that allows assessment of the initial perception of reward in rodents and thereby represents an attractive model for further investigating initial drug effects and dissect reward and locomotor-enhancing responses various drugs (Runegaard et al., 2017a). Chemogenetics has been used widely in various experimental set-ups to further dissect and shed light on behaviors and underlying changes to neural networks related to addiction including acute locomotor response, behavioral sensitization, CPP, and self-administration.

1. Locomotor Sensitization. Locomotor sensitization is a lasting, increased locomotor response observed following repeated, noncontingent administration of psychostimulants. This is believed to reflect the synaptic adaptive changes that occur with repeated drug intake and to be associated with the transition from initial drug use to addiction (Hooks et al., 1991a,b; Piazza et al., 1991; Belin and Deroche-Gamonet, 2012). Not surprisingly, DREADDs have been applied to the DA system to examine the influence of dMSNs and
iMSNs during repeated drug administrations and after drug-induced behavioral sensitization. The results confirmed previous pharmacological and transgenic pieces of evidence on the opposing roles of the indirect and direct MSN pathways (Lobo and Nestler, 2011). Indeed, activation of hM4Di in iMSNs or dMSNs enhanced or attenuated, respectively, behavioral sensitization to amphetamine (Ferguson et al., 2011). Similarly, behavioral sensitization was prevented when Gi-induced signaling was opposed by activating rM3Ds in iMSNs (Farrell et al., 2013).

The findings support that iMSN activity is a driving force underlying behavioral sensitization (Farrell et al., 2013), that is, the experiments indicate that locomotor sensitization, similarly to general locomotor activity (see section II.A.2) depends on a relative output signal from striatal MSNs following repeated drug administration. Interestingly, acute and sensitized responses to psychostimulants seem to be affected differently by the same DREADD manipulations. Activation of hM4Di in VTA neurons strongly inhibited the acute response to cocaine, but this neither prevented the development nor the expression of a sensitized response (Runegaard et al., 2018). On the contrary, hM4Di activation in iMSNs during amphetamine sensitization had no effect on the acute response or during initiation, but prevented expression of behavioral sensitization during a challenge dose 1 week later (Ferguson et al., 2011). This suggests that the responses are driven by separate and to some extent independent mechanisms, possibly segregated by pre- and postsynaptic effects. Moreover, the potential influence and role of coreleased neurotransmitters from heterogeneous populations of midbrain DA neurons must be taken into consideration to understand fully the mechanisms underlying the segregation of these behaviors (Poulin et al., 2018).

2. Reward Perception, Drug-Cue Associations, and Relapse Behaviors. The perception of reward is linked to increases in DA levels, and such increases are considered the common mechanism responsible for the addictive properties of drugs of abuse (Wise and Bozarth, 1985; Di Chiara and Imperato, 1988; Koob and Le Moal, 1997). How the initial increase in DA conveys into adaptive changes that only in a subpopulation of subjects induce the transition from recreational use to addiction is still poorly understood. Based on the fact that females tend to transition faster to addiction and experience greater difficulties remaining abstinent (Becker and Koob, 2016), Calipari et al. (2017) investigated the influence of the estradiol-cycle on VTA signaling and cocaine reward using the CPP paradigm. Their results revealed an estradiol-mediated increase in the activity of VTA DAergic neurons that promoted phosphorylation of DAT, which in turn was proposed to increase the ability of cocaine to inhibit its function. By use of chemogenetics, they moreover obtained data indicating a possible mechanistic link between the 
increased activity of VTA DA neurons, via hM3Dq activation, and the enhanced affinity of cocaine for DAT, subsequent reward processing, and increased CPP (Calipari et al., 2017). In an earlier study, the same research group linked chronic cocaine exposure to dysregulated dMSNs signaling, impaired extinction, and facilitated cocaine-primed reinstatement. Specifically, it was found that hM4Di-mediated inhibition of dMSNs attenuated the strength of drug association and prevented relapse (Calipari et al., 2016).

The bed nucleus of the stria terminalis (BNST) has been implicated in cue-induced cocaine-seeking behaviors, and hM4Di-mediated inhibition of this region suggested that it is involved in ethanol-seeking behaviors (Pina et al., 2015). By using a dual viral chemogenetic approach, it was demonstrated that BNST neurons projecting to the VTA are involved in cue-induced ethanol-seeking behavior, as hM4Di activation in these neurons prevented alcohol CPP (Pina and Cunningham, 2017). Although ethanol has a different pharmacological profile compared with psychostimulants (Vengeliene et al., 2008; Kumar et al., 2009), the influence of DA in mediating the transition to addiction appears similar (Soderpalm and Ericson, 2011; Sulzer, 2011). Interestingly, Cheng et al. (2017) demonstrated an alcohol-induced enhancement of glutamatergic and GABAergic transmission onto dMSNs and iMSNs, respectively. That is, in vivo chemogenetic manipulation of dMSN or iMSN via hM3Dq or hM4Di, mimicking glutamatergic or GABAergic enhancement, respectively, affected alcohol consumption with hM3Dq activation in dMSNs or hM4Di activation in iMSNs promoting alcohol consumption, and oppositely reducing consumption through hM3Dq activation in iMSNs or hM4Di in dMSNs (Cheng et al., 2017). Anatomically distinct functions of the NAc core and shell in alcohol consumption were revealed in a limited access mouse model where alcohol consumption was reduced or increased following hM3Dq or hM4Di stimulation, respectively, of the NAc core but not accumbal shell (Purohit et al., 2018). It has also been observed that activation of KORD in neurons projecting to the NAc from the ventral subiculum decreased context-induced relapse of alcohol self-administration (Marchant et al., 2016a). While the mechanism of action of inhalant drugs of abuse has lagged behind, one recent chemogenetic study revealed an important role of infralimbic cortical projections to the NAc (Wayman and Woodward, 2018). The study described a toluene-induced (i.e., glue sniffing) reduction of excitability in rat infralimbic cortical neurons projecting to the NAc shell and inhibition of toluene-induced place preference after stimulating hM4Dq in this pathway (Wayman and Woodward, 2018).

Chemogenetics has further been employed to obtain evidence that astrocytes modulate the sensitivity and behavioral responses to reinforcing stimuli. Activating
hM3Dq specifically in the astrocytes of the NAc core increased intracranial self-stimulation, as well as reduced motivation to self-administer ethanol after 3 weeks abstinence (Bull et al., 2014). Similar chemogenetic activation of astrocytes expressing hM3Dq was shown to inhibit cue-induced reinstatement of cocaine seeking (Scofield et al., 2015). By the use of glutamate biosensors, Scofield et al. (2015) found that hM3Dq activation in the NAc astrocytes elevated glutamate levels as well as they obtained evidence that this glutamatergic gliotransmission inhibited cue-induced reinstatement of cocaine seeking as a result of activating neurotransmitter release-regulating metabotropic glutamate $2 / 3$ autoreceptors.

The relative output from dMSNs and iMSNs, residing within the NAc, is likely directly to influence relapse since both rM3Ds-mediated stimulation of dMSN and hM4Di-mediated inhibition of iMSNs led to an increase in cue-induced reinstatement of cocaine self-administration (Heinsbroek et al., 2017). This effect was reversed with simultaneous hM4Di-mediated inhibition of ventral pallidum neurons (Heinsbroek et al., 2017). Consistent with these findings, ventral pallidum neurons have been associated with rewardcue pairing and reinstatement, and chemogenetic inhibition of ventral pallidum neurons impaired the ability of rats to acquire sign-tracking behaviors in response to a learned natural reward-associated cue (Chang et al., 2015). Interestingly, different areas of the ventral pallidum seem to regulate different forms of reinstatement. Local application of CNO in VTA of mice expressing hM4Di in either the rostral or caudal ventral pallidum revealed that only cue-induced reinstatement was suppressed when inhibiting rostral ventral pallidum-VTA projecting neurons, whereas caudal ventral pallidum-VTA inhibition had no effect on reinstatement. Conversely, systemic CNO was found to block primed reinstatement in caudal ventral pallidumhM4Di rats, indicating that although caudal ventral pallidum is necessary for cocaine-primed reinstatement, its VTA targets are not essential for this action (Mahler et al., 2014). This suggests a complex contribution of specific subnuclei projections and circuits involving the NAc and ventral pallidum in drug-seeking behaviors. In addition, application of chemogenetics has also pointed to a role of midline thalamic nuclei neurons in drug-seeking behaviors. Interestingly, reducing activity of midline thalamic nuclei neurons by hM4Di stimulation inhibited both cue-induced and cocaine-primed reinstatement, whereas chemogenetic inhibition specifically of efferent projections from the anterior portion of midline thalamic nuclei to the NAc only blocked cocaine-primed reinstatement and enhanced cue-induced reinstatement (Wunsch et al., 2017). Similar to lesions of the NAc, suppressing the activity of accumbal neurons with hM4Di reduced ethanol consumption in a limited access model. In contrast, 
increasing NAc activity with hM3Dq had no effect on ethanol intake (Cassataro et al., 2014). In an alcohol self-administration paradigm, the ventral pallidum was again demonstrated to influence reinstatement with hM4Di or rM3Ds stimulation, resulting in reduced or increased context-induced, as well as prime-induced, reinstatement of extinguished alcohol seeking, respectively (Prasad and McNally, 2016).

Lastly, the medial PFC is an area implicated in compulsive behaviors and has also been associated with relapse behaviors (Kalivas, 2008). Functional dissection of the ventro- and dorsomedial PFC areas has shown anatomically distinct influences on relapse behaviors. For example, the dorsomedial PFC region promoted relapse, while the ventromedial $\mathrm{PFC}$ region regulated extinction-like reductions in cocaine seeking (Peters et al., 2009; Moorman et al., 2015). In cocaine selfadministering rats, hM4Di activation of medial PFC afferents to the NAc had no effect on motivation for taking cocaine during progressive ratio scheduling but increased subsequent drug-seeking behavior during extinction. Furthermore, primed reinstatement was significantly higher in rats that had been treated with CNO during progressive ratio scheduling. Strikingly, CNO treatment prior to a priming injection attenuated this response (Kerstetter et al., 2016). The authors suggested that this indicated a strengthening of the associative processes that linked cocaine with surrounding stimuli, which may seem counterintuitive when dampening medial PFC afferent activity to the NAc. However, the authors further speculated that latestage beta-arrestin-mediated recruitment of mitogenactivated protein kinase/extracellular signal-regulated kinase (ERK) signaling modulated long-term alterations in neuronal plasticity and memory formation (Kerstetter et al., 2016). The specific role of the ventromedial PFC in extinction behaviors was confirmed through a chemogenetic approach aiming at activating ventromedial PFC neurons in a self-administration reinstatement model (Augur et al., 2016). By activating $\mathrm{hM} 3 \mathrm{Dq}$ in ventromedial PFC neurons projecting to the NAc shell, Augur et al. (2016) demonstrated that these neurons played a role following cue-extinction learning, as they observed that cue-, but not prime-induced, reinstatement of cocaine seeking was reduced postextinction. Furthermore, they observed no effect following abstinence where the rats had not been through the "de-learning" phase of the cue-drug pairing (Augur et al., 2016). Altogether, neuronal adaptations seem to occur during extinction, which involves an active process in terms of context extinction memory, making drug-seeking behaviors dependent on ventromedial PFC activity.

3. Compulsive Drug Taking. The resistance to punishment after the acquisition of drug self-stimulation was recently found to be associated with enhanced neural activity in the orbitofrontal cortex. Chemogenetic inhibition of this region reduced this form of compulsivity and prevented persevered self-stimulation despite punishment (Pascoli et al., 2015). Previously, cocaineinduced perseverative behaviors in mice were associated with altered synaptic plasticity in accumbal iMSNs. A potentiation of glutamatergic input to iMSNs was, contrary to potentiation of dMSNs, observed only in mice that showed resilience toward compulsive cocaine seeking. Chemogenetic inhibition of NAc iMSNs enhanced this motivation to obtain cocaine without affecting drug intake (Bock et al., 2013). The data provide a possible input-output link of a natural protective mechanism against compulsive drug intake, with cocaine inducing an enhanced activity of orbitofrontal cortex circuits synapsing onto iMSNs within the NAc.

Summarized, the studies described above on the role of the DA system in addiction reveal how certain neuronal populations and regions already associated with the DA reward circuitry system can influence addictive behaviors. Although several findings are not surprising as they support previous hypotheses, they provide a novel and unique spatial-selective map. Since regions and specific neuronal populations can be linked together, this combination reveals an immensely complex reward system, with pathways projecting from multiple brain regions that either directly or indirectly regulate prime regions of DA transmission including the VTA and NAc.

\section{Mood, Nociception, and Cognition}

Anxiety and depression are among the most common neuropsychiatric disorders, but the underlying neurobiology is poorly understood. Although 5-HT dysfunction is classically associated with mood disorders, the possible contribution of DAergic neurons in mood regulation has been examined using DREADDs. Zhong et al. (2014) induced an anxiogenic-like and depressivelike phenotype by selectively deleting expression of $\mathrm{Cdk} 5$, a kinase involved in the phosphorylation of $\mathrm{TH}$, in the VTA of mice. This deletion caused reduced TH phosphorylation and VTA cAMP levels and NAc DA release. Selective rM3Ds activation of VTA DA neurons in this genetically modified mouse strain restored $\mathrm{TH}$ phosphorylation and DA levels, as well as rescued the behavioral deficits induced by deletion of the kinase (Zhong et al., 2014). DAergic neurons were also implicated in depressive-like behaviors under systemic inflammation conditions. When discomfort associated with a systemically induced inflammation state was investigated in the CPP paradigm, mice demonstrated an aversion to the inflammation-paired chamber and preferred the neutral chamber (Fritz et al., 2016). Chemogenetic activation of hM3Dq-expressing mice in midbrain DAergic neurons abolished this systemic inflammation place aversion, suggesting that this aversion is mediated via or at least integrated into midbrain DAergic neurotransmission. 
Other studies have focused on the regulation of DAergic neurons under pain conditions. Chemogenetic suppression of VTA DAergic activity offset the phenomenon of exercise-induced hypoalgesia in partially sciatic nerve-ligated mice (Wakaizumi et al., 2016). Furthermore, specific hM3Dq activation of DAergic neurons in the ventral periaqueductal gray/dorsal raphe neurons was found to induce an anti-nociceptive effect in the hotplate, but not the tail flick assay, proposing that DA released from the periaqueductal gray/dorsal raphe is associated with supraspinal, but not spinal, nociception (Li et al., 2016).

DA was also shown to play a crucial role in fear learning, and therefore anxiety disorders and posttraumatic stress disorder (Lee et al., 2016; Bouchet et al., 2018; Mayer et al., 2018). Yet how DAergic neurons modulate these processes is unclear. Chemogenetic tools have facilitated investigations into this subject area, with hM4Di-mediated inhibition of a specific part of the mesolimbic system, projecting from the posterior medial VTA to the NAc shell, blocking relief learning following an aversive electrical stimulus cue (Mayer et al., 2018). There is ever growing data implicating the nigrostriatal pathway in this form of emotion since hM3Dq-mediated activation of SN DAergic neurons improved fear extinction memories and prevented future fear renewals (Bouchet et al., 2018). These early data propose an important role of DAergic pathways in the processing of signals predicting fear and relief, and therefore may represent a potential target for improving extinction-based therapies in anxiety and traumarelated disorders (Lee et al., 2016).

DA is known to play key roles in PFC-mediated cognition with system deficits apparent in ADHD and schizophrenia (Arnsten and Pliszka, 2011). To elucidate the cellular mechanisms governing $\mathrm{D}_{2} \mathrm{R}$-mediated enhancement of PFC pyramidal neuron excitability, each CNO-sensitive DREADD was probed in an in vitro electrophysiological set up. Strikingly, the results showed that only Gs-DREADD stimulation of the medial PFC mimicked this phenomenon implicated in schizophrenia (Robinson and Sohal, 2017). It is not only the higher cortical areas where DREADDs have been applied to study DA-regulated cognition. The importance of $\mathrm{D}_{1} \mathrm{Rs}$ located in the lateral nucleus of the cerebellum was demonstrated using hM4Di-mediated cerebellar stimulation in $\mathrm{D}_{1} \mathrm{R}$-Cre mice to induce deficits on working memory and prepulse inhibition of the acoustic startle response (Locke et al., 2018).

The five-choice serial reaction time task assesses attentional, impulsive, and motivational control in rodents, and it is well-known that the DAergic system is centrally implicated in regulating all these behaviors, as also previously reviewed (Robbins, 2002; Jørgensen et al., 2017). In short, food-restricted subjects must wait and attend to a brief light stimulus in one of five apertures to receive a reward. The number of premature responses, before the light appears, represents a measure of impulsivity, while incorrect or omitted responses are considered a measure of attention impairments. Interestingly, activation of hM3Dq in the rat VTA and SN pars compacta was shown to cause an increase in omitted responses, while only SN pars compacta activation decreased the number of correctly responded trials and mean correct latency (Boekhoudt et al., 2017b). As no effect on premature responses was observed, the authors suggested that increased midbrain DA neuronal activity caused attentional, but not impulsive, deficits.

\section{Discussion and Future Directions}

Summarized, it seems very clear that use of DREADDs in the area of DA research has advanced substantially our understanding of the DA system and led to many new discoveries. Indeed, the DAergic system is complex, consisting of a heterogeneous population of DA neurons and output cells that are intermingled and difficult to target in behavioral studies. By providing the spatial specificity that standard pharmacologic interventions lack, the chemogenetic toolbox has shown its potential to dissect the DA system and enlighten its influence on behavior. The DREADDs technique allows for relatively noninvasive regulation of neuronal signaling and depends largely on the intrinsic ability of neurons to regulate their own activity, as DREADDs work through endogenous intracellular signaling mechanisms. Once a chemogenetic receptor is expressed in neuronal populations/projections of interest, systemic administration of the inert ligand, CNO/clozapine or SalB, permits transient and repeated perturbations of neural activity. This can even be accomplished bilaterally in large or elongated brain structures, which can be extremely difficult with microinjections of pharmacologic compounds or other molecular-genetic approaches such as optogenetics (Smith et al., 2016).

Despite its far-reaching usefulness and potential, the DREADDs technology is still in its infancy, and we are only beginning to realize the true nature of this toolbox's contents. However, as any other technique, chemogenetic interventions are not without limitations and challenges, and a lot of effort should be put into understanding the actual effects of injecting, expressing, and activating the various DREADDs, as well as possible adaptive changes occurring due to repeated activation.

\section{A. Designer Receptors Exclusively Activated By Designer Drugs Effects in Theory and in Reality}

1. Designer Receptors Exclusively Activated By Designer Drugs Expression-The Meaning of Inertness. The chemogenetic toolbox contains mutated GPCRs that are inert until application of their agonist (see 
Fig. 1). Supposing that the DREADD agonists CNO and SalB are also inert and do not target endogenous receptors, their use provides the neuroscience field with, in theory, an ideal system for pharmacological remote control of any neuronal population of interest. However, there are no guarantees that this inertness applies in reality, and, as mentioned in the introduction (section I.2), recent evidence has emerged, indicating that at least under some circumstances in certain systems, extra caution should be exerted when interpreting chemogenetic experimental data (Maclaren et al., 2016; Gomez et al., 2017; Padovan-Hernandez and Knackstedt, 2018). CNO was previously reported to back-metabolize to clozapine in humans, nonhuman primates, and guinea pigs, but has generally been considered inert in rats and mice (Jann et al., 1994; Chang et al., 1998; Guettier et al., 2009; Loffler et al., 2012). More recently, however, MacLaren et al. (2016) described multiple dosedependent effects in vivo in rats as a consequence of the back-metabolism of CNO to its bioactive parent compounds clozapine and $N$-desmethylclozapine. Still, at CNO doses most commonly applied ( 1 to $2 \mathrm{mg} / \mathrm{kg}$ ), there were no measurable effects on basal locomotion, nor amphetamine-induced DA release or hyperactivity. The acoustic startle reflex was the only tested measure that was affected by 1 or $2 \mathrm{mg} / \mathrm{kg}$ CNO (Maclaren et al., 2016). In the last decade, DREADDs have been applied in hundreds of laboratories to study various different neuronal circuits reporting no effects of CNO in nonDREADD-expressing mice. Accordingly, the concerns regarding the use of DREADDs to functional studies in vivo seem to be replaced with prudent caution and refinement (Mahler and Aston-Jones, 2018). To what extent SalB is inert remains to be determined. As a general recommendation, researchers should include and carefully plan control studies to exclude potential undesirable effects caused by DREADD ligands themselves.

So far, there have been no reports of constitutive activity of DREADDs expressed within neurons. Nonetheless, logically such activity should correlate with not just the number of DREADDs but also the endogenous signal transduction machinery, which varies between different populations of cells and neurons. Some constitutive activity of the rM3Ds DREADD in transfected cells has been reported and possibly explains the modest basal phenotype described in pancreatic beta cells (Guettier et al., 2009). Hence, chemogenetic receptors may not have profound basal activity in neuronal systems tested, but it cannot be ruled out that they can present with constitutive activity in certain cells of the CNS or when expressed by certain approaches. Low levels of constitutive activity might not cause a detectable phenotype right away but instead induce subtle alterations in homeostasis and long-term behaviors.

Another important issue is potential concerns relating to the method used to introduce DREADDs into neurons and how this might differentially affect the function of the cells. It has, for example, been shown in optogenetic studies that the route of administration of the exact same opsin using identical AAV vector backbone, but encapsulated with various capsids (i.e., serotypes $1,5,8,9$ ), affected the efficacy of neuronal activity differently (Jackman et al., 2014). If this also applies to DREADDs, then even the choice of AAV serotype can determine how neurons respond to DREADD ligands. While such administration-related phenomena might not be a concern for interpreting one single DREADD study, it could impede comparisons between studies across the same scientific objectives in which the expression of DREADDs in identical cells were obtained by different viral serotypes and/or transgenic animals.

2. Interpretation of Activating Designer Receptors Exclusively Activated By Designer Drugs with Clozapine $\mathrm{N}$-Oxide. Interpretation of chemogenetic manipulations affecting behavior is often condensed to activation or silencing of a specific neuron population/projection by hM3Dq/rM3Ds and/or hM4Di/KORD, respectively, but this is possibly an extreme simplification of what CNO actually does to neurons expressing DREADDs. Firstly, the effect of activating a certain DREADD may differ between neurons and even subpopulations of neurons, depending on the intracellular signal machinery, as mentioned above. Hence, the effect of activating hM4Di in different populations of neurons or even within the same neurons at different times can be quite different. For example, the activation of neurons by hM4Di inhibits presynaptic firing by hyperpolarizing neurons via GIRK (Roth, 2016), but some neurons may possess low expression of GIRK, and, consequently, the effect of CNO in these neurons may be negligible or dominated by other downstream mediators. In addition, GIRK levels may change because of previous drug exposure. Both cocaine and methamphetamine have been reported to inhibit midbrain GIRKmediated currents in DAergic brain neurons for up to 2 weeks post-psychostimulant administration (Kobayashi et al., 2007; Padgett et al., 2012; Sharpe et al., 2014). This psychostimulant-related effect on GIRK is important to consider when utilizing chemogenetic tools, especially $\mathrm{hM} 4 \mathrm{Di}$, in DA-based research, given how psychostimulants alter DAergic neural circuitry and contribute to locomotion and reward-related processes. Recently, we demonstrated that following withdrawal of repeated cocaine, hM4Distimulation of VTA DA neurons no longer influenced cocaine-induced locomotion (Runegaard et al., 2018) Therefore, it will be important to investigate further whether and to what degree various drugs, including cocaine and amphetamine, affect the efficacy of chemogenetic modulation on neuronal activity and behavior, including how DREADD expression affects ligand-receptor binding interactions. 
3. Considerations for Repeated Activation of Designer Receptors Exclusively Activated By Designer Drugs. CNO-induced activation of DREADDs depends on endogenous GPCR signaling cascades, and although these designer receptors are always available and should not desensitize, downstream effectors may limit the neuronal effect upon repeated activation (e.g., GIRK desensitization) (Roth, 2016). Due to the high expression levels of chemogenetic receptors, and the subsequent amount of receptor reserve, substantial desensitization by CNO or SalB causing a blunted or absent effect is obviously less likely. However, this is an issue that has yet not been satisfactorily addressed (Roman et al., 2016; Roth, 2016). So far, it has been shown that mice expressing hM4Di in the dorsomedial striatum or NAc developed a tolerance toward CNO following 2 weeks of chronic administration. However, that tolerance disappeared $48 \mathrm{~h}$ after chronic administration stopped (Carvalho Poyraz et al., 2016). Bellocchio et al. (2016) also found that chronic activation of hM3Dq with a high CNO dose of $10 \mathrm{mg} / \mathrm{kg}$ altered behavioral responses and induced cell death in vivo, possibly due to the unnatural, intense signal cascades activated by $\mathrm{CNO}$, which caused neuronal damage. They discovered that chronic hM3Dq activation in iMSN caused enhanced locomotor activity and neuronal death via the c-Jun $\mathrm{N}$-terminal kinase pathway (Bellocchio et al., 2016). Accordingly, it is important when designing long-lasting DREADD experiments to exclude possible adaptations caused by repeated administration of DREADD ligands.

4. Can Selective Dopamine Expression Be Achieved? As previously mentioned, one major advantage of DREADDs, but not limited to this specific technology, is the spatial selectivity by which the manipulation can be performed. Although the theoretical foundation of selective DREADD expression by Cre-dependency and/or promoter-regulated expression appears solid, practical issues such as leaky expression of Cre and concerns regarding promoter specificity may hamper the actual specificity and confound interpretations. In addition, the transgenic Cre-driver lines applied to specify DREADDs expression have not all been validated, which could also confound interpretation of DREADDs data. Thus, it seems important that researchers validate their transgenic Cre-driver line before proceeding with chemogenetic manipulationsin particular when WT littermates (non-DREADD expressing) are included as a control for CNO's own effects. Importantly, we recently characterized the commonly used TH-Cre driver mouse line (Savitt et al., 2005) and found neither alterations in DA system-related function nor in behavior (Runegaard et al., 2017b).

Recent studies have revealed a higher degree of structural, as well as functional heterogeneity, in the DA system than previously assumed. This calls for increased anatomic specificity when manipulating specific DA neurons and projections to provide reliable and accurate interpretations. Indeed, concerns have been raised regarding the use of a single gene marker to define the DAergic phenotype (Björklund and Dunnett, 2007; Apuschkin et al., 2015; Lammel et al., 2015; Stuber et al., 2015), and the DAT- and TH-Cre driver lines used to specify expression to DAergic neurons may target different but overlapping populations of DAergic neurons. Furthermore, recent evidence suggests that effector gene expression in transgenic TH-Cre mice is not limited to DAergic neurons but also seen ectopically such as in TH-transcript positive GABAergic VTA neurons projecting to the lateral habenula (Lammel et al., 2015; Stuber et al., 2015). This knowledge emphasizes the need for an updated characterization of the DA phenotype, or rather a dissection of the different phenotypes of this heterogeneous population of neurons. Specifically, it might be necessary to redefine the hallmarks of a DAergic neuron, considering whether it is defined by its synthesis, release, and/or reuptake mechanisms (Berke, 2018). It is, moreover, essential to achieve a better understanding of the DAergic subpopulations coreleasing other neurotransmitters such as glutamate (Morales and Root, 2014) and GABA (Barker et al., 2016) to guide us in our understanding of DAergic neuronal and projection patterns.

5. Can Designer Receptors Exclusively Activated By Designer Drugs Help Dissect the Dopaminergic Pathways? As emphasized above, a fair interpretation of the chemogenetic manipulations relies on the thorough validation of targeted neurons, projections, and an understanding of their characteristics. Independent of the definition of DAergic neurons, refinements of the chemogenetic technique are also important. These include improvements in projection-specific expression by the use of retrogradely transported viral vectors (e.g., CAV-2-Cre) (Boender et al., 2014), recombinant AAVs carrying tissue- or cell-specific promoters (Gompf et al., 2015), or by double-recombination approaches through the introduction of an additional recombinase, such as Flp (Stamatakis et al., 2013). This latter approach could be even more sophisticated if introduced under an activity-dependent promoter, e.g., mediated by C-fos activation (Garner et al., 2012; Kawashima et al., 2014; Roy et al., 2016). Additionally, the temporal and spatial resolution can be improved further via photo-caging DREADDs or creating DREADD antagonists, which could become highly useful and emphasize the continuous development and refinement of the DREADD technology (Roth, 2016). Of further interest, refinement of viral genetic tracing techniques has recently allowed systematic mapping of the input-output circuitry of neuronal nuclei (Beier et al., 2015; Schwarz et al., 2015; Cardozo Pinto and Lammel, 2018). Beier et al. (2015) demonstrated that VTA DA neurons projecting to different forebrain regions exhibited specific biases in 
their input selection, and VTA DA neurons projecting to the lateral and medial NAc innervated largely nonoverlapping striatal targets, with the latter also sending extrastriatal axon collaterals.

\section{B. Have Chemogenetic Studies Advanced Our Understanding of Dopamine-Related Behaviors?}

While the chemogenetic toolbox has facilitated studies previously not possible, it is evident that some of the results obtained by application of chemogenetics do not provide true novel insights but rather confirm what has previously been shown by lesion studies and by use of transgenic or pharmacologic approaches. However, as described in this review, the improved specificity of the chemogenetic manipulations has in many cases enabled much more refined conclusions in terms of spatial and specific influences of distinct neuronal populations on behavioral outputs. We believe accordingly that future chemogenetic studies have the potential to significantly further advance our understanding of the complex role of DA in regulating the multiple behaviors discussed here. Indeed, the wide use of chemogenetics in laboratories all over the world directly reflects the vast potential of the technology to progress our understanding of neuronal signaling and behavior. Nonetheless, when interpreting chemogenetic data with a wish to conclude about DA-related behaviors, it is highly important to consider the immense heterogeneity of the DA system. Not least, the still poorly understood importance of coreleased neurotransmitters, such as GABA and glutamate, must be taken into account (Poulin et al., 2018). Moreover, and as also mentioned earlier, it has to be considered that when chemogenetically manipulating, e.g., MSNs, the outcome might not necessarily solely reflect DA transmission, because neurons receive metabotropic input from multiple GPCRs and not only $\mathrm{G}$ protein-coupled DRs. In context of these challenges, it will be highly important to combine the specificity by which DREADDs can be expressed and activated with advanced techniques assessing neurotransmitter release, activity, and dynamics, such as the recently published DA biosensors (Patriarchi et al., 2018; Sun et al., 2018) or two-photon excitation microscopy technologies (Svoboda and Yasuda, 2006). Such approaches should indeed enable better comprehension of the multifaceted action governed by DA and its circuitry in the near future.

\section{Future Directions}

Besides the use of DREADDs in biologic and disease research, this technique possesses tremendous potential in translational research and clinical therapies (Roth, 2016). As well as developing multiple designer receptors and agonists, the fast-paced DREADDs field has dissected the mesocorticolimbic network with positron emission tomography (Michaelides et al., 2013) and functional magnetic resonance imaging (Roelofs et al., 2017) and has already been used in nonhuman primates (Eldridge et al., 2016; Grayson et al., 2016). While the idea of gene therapy and thereby expressing DREADDs in humans may not be a major issue, it is the ligand CNO that arouses most worry because of the back-metabolization of CNO to clozapine in several species, potentially limiting its use in patients. However, the newer KORD variant is activated by a different ligand, SalB, which has not yet been associated with such issues (Vardy et al., 2015). In addition, structure-activity relationship studies were applied to develop alternative ligands for activating DREADDs, leading to the identification of a new potent $\mathrm{hM} 3 \mathrm{Dq}$ agonist that is neither back-metabolized nor activating endogenous receptors (Chen et al., 2015). Development of more sophisticated DREADD variants will thereby probably allow for the use of DREADDs in humans in the future (Urban and Roth, 2015; Smith et al., 2016).

\section{Conclusion}

A plethora of novel techniques has become available within the field of neuroscience in recent years. Together these have revolutionized neuroscience and dramatically improved our understanding of complex processes taking place in the brain and how such processes might change during disease states. One technique of particular interest is chemogenetics that, by allowing pharmacological remote control of neuronal activity, have opened up for entirely new possibilities for dissecting neuronal circuits and deciphering the role of diverse neuronal populations. Chemogenetics has already been extensively used for studying the DA system, and, as reviewed here, multiple different components of the DA system, including DA-releasing neurons, striatal postsynaptic MSNs conveying DAergic signals, and other neural regions/substances synapsing onto the DA neurons, have been targeted by DREADDs to investigate regulation of discrete and multiple behavioral functions. One important lesson is that we should learn and appreciate that behavior is not simply driven by just one single neuronal population or projection pattern, but rather determined by a tightly regulated integration of multiple neuronal inputs. Indeed, behavior is complex and should not be simplified to a binary constituent taking place in response to "activation" or "inhibition" of specific neurons.

The major advancement of chemogenetics, compared with traditional pharmacological interventions, has been the increased anatomic specificity by which the manipulations can be undertaken and thereby dissect not just a biologic heterogeneous population of DA neurons, but also an anatomically important complex projection system. There are no doubts that the potential of chemogenetics is huge, but as with any other technique, there are concerns, some of which need to be further addressed. These include how to target a 
heterogeneous DAergic population, the dependency of DREADDs-related effects on endogenous GPCR signaling machinery (which may differ between different neuronal subpopulations), expression-induced adaptations, as well as possible alterations in DREADDs expression and/or adaptive cellular responses with repeated or chronic CNO administration. It should nonetheless be appreciated that the chemogenetic technique is still at a relatively early stage, and there is no reason to believe that many of these obstacles cannot be addressed and the technique further developed. With the progress of the CRISPR technology (Wang et al., 2016), one might even consider that DREADDs-like manipulations of any endogenous receptor/transporter of interests soon might become part of the commonly used methodological repertoire. In relation to direct translational application of the DREADD technology, searches for new ligands (Chen et al., 2015; Roth, 2016) and development of new designer receptor variants activated by other ligands (e.g., KORD) (Vardy et al., 2015), may overcome the problem of extensive metabolism of the activating ligand CNO in primates, and thus, chemogenetic therapy to treat neurologic and psychiatric disorders may not be an unrealistic or distant goal.

\section{Authorship Contributions}

Wrote or contributed to the writing of the manuscript: Runegaard, Fitzpatrick, Woldbye, Andreason, Sørensen, and Gether.

\section{References}

Alcacer C, Andreoli L, Sebastianutto I, Jakobsson J, Fieblinger T, and Cenci MA (2017) Chemogenetic stimulation of striatal projection neurons modulates responses to Parkinson's disease therapy. J Clin Invest 127:720-734.

Aldrin-Kirk P, Heuer A, Rylander Ottosson D, Davidsson M, Mattsson B, and Björklund T (2018) Chemogenetic modulation of cholinergic interneurons reveals their regulating role on the direct and indirect output pathways from the striatum. Neurobiol Dis 109 (Pt A):148-162.

Aldrin-Kirk P, Heuer A, Wang G, Mattsson B, Lundblad M, Parmar M, and Björklund T (2016) DREADD modulation of transplanted DA neurons reveals a novel Parkinsonian dyskinesia mechanism mediated by the serotonin 5-HT6 receptor. Neuron 90:955-968.

Alexander GM, Rogan SC, Abbas AI, Armbruster BN, Pei Y, Allen JA, Nonneman RJ, Hartmann J, Moy SS, Nicolelis MA, et al. (2009) Remote control of neuronal activity in transgenic mice expressing evolved G protein-coupled receptors. Neuron 63:27-39.

Apuschkin M, Stilling S, Rahbek-Clemmensen T, Sørensen G, Fortin G, Herborg Hansen F, Eriksen J, Trudeau LE, Egerod K, Gether U, et al. (2015) A novel dopamine transporter transgenic mouse line for identification and purification of midbrain dopaminergic neurons reveals midbrain heterogeneity. Eur $J$ Neurosci 42:2438-2454.

Arias-Carrión O and Pŏppel E (2007) Dopamine, learning, and reward-seeking behavior. Acta Neurobiol Exp (Warsz) 67:481-488.

Armbruster BN, Li X, Pausch MH, Herlitze S, and Roth BL (2007) Evolving the lock to fit the key to create a family of G protein-coupled receptors potently activated by an inert ligand. Proc Natl Acad Sci USA 104:5163-5168.

Arnsten AFT and Pliszka SR (2011) Catecholamine influences on prefrontal cortical function: relevance to treatment of attention deficit/hyperactivity disorder and related disorders. Pharmacol Biochem Behav 99:211-216.

Augur IF, Wyckoff AR, Aston-Jones G, Kalivas PW, and Peters J (2016) Chemogenetic activation of an extinction neural circuit reduces cue-induced reinstatement of cocaine seeking. J Neurosci 36:10174-10180.

Bäckman CM, Malik N, Zhang Y, Shan L, Grinberg A, Hoffer BJ, Westphal H, and Tomac AC (2006) Characterization of a mouse strain expressing Cre recombinase from the $3^{\prime}$ untranslated region of the dopamine transporter locus. Genesis 44:383-390.

Baik J-H (2013) Dopamine signaling in reward-related behaviors. Front Neural Circuits 7:152

Balfour ME, Yu L, and Coolen LM (2004) Sexual behavior and sex-associated environmental cues activate the mesolimbic system in male rats. Neuropsychopharmacology 29:718-730.

Barker DJ, Root DH, Zhang S, and Morales M (2016) Multiplexed neurochemical signaling by neurons of the ventral tegmental area. J Chem Neuroanat 73:33-42.
Barker RA, Barrett J, Mason SL, and Björklund A (2013) Fetal dopaminergic transplantation trials and the future of neural grafting in Parkinson's disease. Lancet Neurol 12:84-91.

Barter JW, Castro S, Sukharnikova T, Rossi MA, and Yin HH (2014) The role of the substantia nigra in posture control. Eur J Neurosci 39:1465-1473.

Bassareo V and Di Chiara G (1999) Differential responsiveness of dopamine transmission to food-stimuli in nucleus accumbens shell/core compartments. Neuroscience 89:637-641.

Beaulieu JM, Espinoza S, and Gainetdinov RR (2015) Dopamine receptors - IUPHAR review 13. Br J Pharmacol 172:1-23.

Beaulieu J-M and Gainetdinov RR (2011) The physiology, signaling, and pharmacology of dopamine receptors. Pharmacol Rev 63:182-217.

Beaulieu JM, Sotnikova TD, Marion S, Lefkowitz RJ, Gainetdinov RR, and Caron MG (2005) An Akt/beta-arrestin 2/PP2A signaling complex mediates dopaminergic neurotransmission and behavior. Cell 122:261-273.

Beaulieu JM, Sotnikova TD, Yao WD, Kockeritz L, Woodgett JR, Gainetdinov RR, and Caron MG (2004) Lithium antagonizes dopamine-dependent behaviors mediated by an AKT/glycogen synthase kinase 3 signaling cascade. Proc Natl Acad Sci USA 101:5099-5104.

Beaulieu JM, Tirotta E, Sotnikova TD, Masri B, Salahpour A, Gainetdinov RR, Borrelli E, and Caron MG (2007) Regulation of Akt signaling by D2 and D3 dopamine receptors in vivo. $J$ Neurosci 27:881-885.

Becker JB and Koob GF (2016) Sex differences in animal models: focus on addiction. Pharmacol Rev 68:242-263.

Beier KT, Steinberg EE, DeLoach KE, Xie S, Miyamichi K, Schwarz L, Gao XJ, Kremer EJ, Malenka RC, and Luo L (2015) Circuit architecture of VTA dopamine neurons revealed by systematic input-output mapping. Cell 162:622-634.

Belin D and Deroche-Gamonet V (2012) Responses to novelty and vulnerability to cocaine addiction: contribution of a multi-symptomatic animal model. Cold Spring Harb Perspect Med 2:a011940.

Bellocchio L, Ruiz-Calvo A, Chiarlone A, Cabanas M, Resel E, Cazalets J-R, Blázquez C, Cho YH, Galve-Roperh I, and Guzmán M (2016) Sustained Gq-protein signaling disrupts striatal circuits via JNK. $J$ Neurosci 36:10611-10624.

Beloate LN, Omrani A, Adan RA, Webb IC, and Coolen LM (2016) Ventral tegmental area dopamine cell activation during male rat sexual behavior regulates neuroplasticity and D-amphetamine cross-sensitization following sex abstinence. $J$ Neurosci 36:9949-9961.

Ben-Shaanan TL, Azulay-Debby H, Dubovik T, Starosvetsky E, Korin B, Schiller M, Green NL, Admon Y, Hakim F, Shen-Orr SS, et al. (2016) Activation of the reward system boosts innate and adaptive immunity. Nat Med 22:940-944.

Berke JD (2018) What does dopamine mean? Nat Neurosci 21:787-793.

Berrios J, Stamatakis AM, Kantak PA, McElligott ZA, Judson MC, Aita M, Rougie M, Stuber GD, and Philpot BD (2016) Loss of UBE3A from TH-expressing neurons suppresses GABA co-release and enhances VTA-NAc optical self-stimulation. Nat Commun 7:10702.

Björklund A and Dunnett SB (2007) Dopamine neuron systems in the brain: an update. Trends Neurosci 30:194-202.

Blum ID, Zhu L, Moquin L, Kokoeva MV, Gratton A, Giros B, and Storch KF (2014) A highly tunable dopaminergic oscillator generates ultradian rhythms of behavioral arousal. eLife 3:e05105.

Bobadilla AC, Garcia-Keller C, Heinsbroek JA, Scofield MD, Chareunsouk V, Monforton C, and Kalivas PW (2017) Accumbens mechanisms for cued sucrose seeking. Neuropsychopharmacology 42:2377-2386.

Bock R, Shin JH, Kaplan AR, Dobi A, Markey E, Kramer PF, Gremel CM, Christensen $\mathrm{CH}$, Adrover MF, and Alvarez VA (2013) Strengthening the accumbal indirect pathway promotes resilience to compulsive cocaine use. Nat Neurosci 16:632-638.

Boekhoudt L, Omrani A, Luijendijk MCM, Wolterink-Donselaar IG, Wijbrans EC, van der Plasse G, and Adan RAH (2016) Chemogenetic activation of dopamine neurons in the ventral tegmental area, but not substantia nigra, induces hyperactivity in rats. Eur Neuropsychopharmacol 26:1784-1793.

Boekhoudt L, Roelofs TJM, de Jong JW, de Leeuw AE, Luijendijk MCM, WolterinkDonselaar IG, van der Plasse G, and Adan RAH (2017a) Does activation of midbrain dopamine neurons promote or reduce feeding? Int J Obes (Lond) 41:1131-1140.

Boekhoudt L, Voets ES, Flores-Dourojeanni JP, Luijendijk MC, Vanderschuren LJ, and Adan RA (2017b) Chemogenetic activation of midbrain dopamine neurons affects attention, but not impulsivity, in the five-choice serial reaction time-task in rats. Neuropsychopharmacology 42:1315-1325.

Boekhoudt L, Wijbrans EC, Man JHK, Luijendijk MCM, de Jong JW, van der Plasse G, Vanderschuren LJMJ, and Adan RAH (2018) Enhancing excitability of dopamine neurons promotes motivational behaviour through increased action initiation. Eur Neuropsychopharmacol 28:171-184

Boender AJ, de Jong JW, Boekhoudt L, Luijendijk MCM, van der Plasse G, and Adan RAH (2014) Combined use of the canine adenovirus-2 and DREADD-technology to activate specific neural pathways in vivo. PLoS One 9:e95392.

Bouabid S and Zhou FM (2018) Cyclic AMP-producing chemogenetic activation of indirect pathway striatal projection neurons and the downstream effects on the globus pallidus and subthalamic nucleus in freely moving mice. $J$ Neurochem 145 : 436-448.

Bouchet CA, Miner MA, Loetz EC, Rosberg AJ, Hake HS, Farmer CE, Ostrovskyy M, Gray N, and Greenwood BN (2018) Activation of nigrostriatal dopamine neurons during fear extinction prevents the renewal of fear. Neuropsychopharmacology 43 : $665-672$

Bull C, Freitas KCC, Zou S, Poland RS, Syed WA, Urban DJ, Minter SC, Shelton KL Hauser KF, Negus SS, et al. (2014) Rat nucleus accumbens core astrocytes modulate reward and the motivation to self-administer ethanol after abstinence. Neuropsychopharmacology 39:2835-2845.

Calipari ES, Bagot RC, Purushothaman I, Davidson TJ, Yorgason JT, Peña CJ, Walker DM, Pirpinias ST, Guise KG, Ramakrishnan C, et al. (2016) In vivo imaging identifies temporal signature of D1 and D2 medium spiny neurons in cocaine reward. Proc Natl Acad Sci USA 113:2726-2731. 
Calipari ES, Juarez B, Morel C, Walker DM, Cahill ME, Ribeiro E, Roman-Ortiz C, Ramakrishnan C, Deisseroth K, Han M-H, et al. (2017) Dopaminergic dynamics underlying sex-specific cocaine reward. Nat Commun 8:13877.

Cardozo Pinto DF and Lammel S (2018) Viral vector strategies for investigating midbrain dopamine circuits underlying motivated behaviors. Pharmacol Biochem Behav 174:23-32.

Carlsson A and Waldeck B (1958) A fluorimetric method for the determination of dopamine (3-hydroxytyramine). Acta Physiol Scand 44:293-298.

Carter ME, Soden ME, Zweifel LS, and Palmiter RD (2013) Genetic identification of a neural circuit that suppresses appetite. Nature 503:111-114.

Carvalho Poyraz F, Holzner E, Bailey MR, Meszaros J, Kenney L, Kheirbek MA, Balsam PD, and Kellendonk C (2016) Decreasing striatopallidal pathway function enhances motivation by energizing the initiation of goal-directed action. $J$ Neurosci 36:5988-6001.

Cassataro D, Bergfeldt D, Malekian C, Van Snellenberg JX, Thanos PK, Fishell G, and Sjulson L (2014) Reverse pharmacogenetic modulation of the nucleus accumbens reduces ethanol consumption in a limited access paradigm. Neuropsychopharmacology 39:283-290.

Chan KY, Jang MJ, Yoo BB, Greenbaum A, Ravi N, Wu WL, Sánchez-Guardado L, Lois C, Mazmanian SK, Deverman BE, et al. (2017) Engineered AAVs for efficient noninvasive gene delivery to the central and peripheral nervous systems. Nat Neurosci 20:1172-1179.

Chang SE, Todd TP, Bucci DJ, and Smith KS (2015) Chemogenetic manipulation of ventral pallidal neurons impairs acquisition of sign-tracking in rats. Eur $J$ Neurosci 42:3105-3116.

Chang WH, Lin SK, Lane HY, Wei FC, Hu WH, Lam YWF, and Jann MW (1998) Reversible metabolism of clozapine and clozapine N-oxide in schizophrenic patients. Prog Neuropsychopharmacol Biol Psychiatry 22:723-739.

Chen X, Choo H, Huang XP, Yang X, Stone O, Roth BL, and Jin J (2015) The first structure-activity relationship studies for designer receptors exclusively activated by designer drugs. ACS Chem Neurosci 6:476-484.

Chen Y, Xiong M, Dong Y, Haberman A, Cao J, Liu H, Zhou W, and Zhang S-C (2016) Chemical control of grafted human PSC-derived neurons in a mouse model of Parkinson's disease. Cell Stem Cell 18:817-826.

Cheng Y, Huang CCY, Ma T, Wei X, Wang X, Lu J, and Wang J (2017) Distinct synaptic strengthening of the striatal direct and indirect pathways drives alcohol consumption. Biol Psychiatry 81:918-929.

Cho JR, Treweek JB, Robinson JE, Xiao C, Bremner LR, Greenbaum A, and Gradinaru V (2017) Dorsal raphe dopamine neurons modulate arousal and promote wakefulness by salient stimuli. Neuron 94:1205-1219.e8.

Chu HY, McIver EL, Kovaleski RF, Atherton JF, and Bevan MD (2017) Loss of hyperdirect pathway cortico-subthalamic inputs following degeneration of midbrain dopamine neurons. Neuron 95:1306-1318.e5.

Chuhma N, Zhang H, Masson J, Zhuang X, Sulzer D, Hen R, and Rayport S (2004) Dopamine neurons mediate a fast excitatory signal via their glutamatergic synapses. J Neurosci 24:972-981.

Conklin BR, Hsiao EC, Claeysen S, Dumuis A, Srinivasan S, Forsayeth JR, Guettier JM, Chang WC, Pei Y, McCarthy KD, et al. (2008) Engineering GPCR signaling pathways with RASSLs. Nat Methods 5:673-678.

Damsma G, Pfaus JG, Wenkstern D, Phillips AG, and Fibiger HC (1992) Sexual behavior increases dopamine transmission in the nucleus accumbens and striatum of male rats: comparison with novelty and locomotion. Behav Neurosci 106: 181-191.

dela Peña I, Gevorkiana R, and Shi W-X (2015) Psychostimulants affect dopamine transmission through both dopamine transporter-dependent and independent mechanisms. Eur J Pharmacol 764:562-570.

Delfs JM, Schreiber L, and Kelley AE (1990) Microinjection of cocaine into the nucleus accumbens elicits locomotor activation in the rat. $J$ Neurosci 10:303-310.

Dell'Anno MT, Caiazzo M, Leo D, Dvoretskova E, Medrihan L, Colasante G, Giannelli S, Theka I, Russo G, Mus L, et al. (2014) Remote control of induced dopaminergic neurons in parkinsonian rats. $J$ Clin Invest 124:3215-3229.

Deminiere JM, Piazza PV, Le Moal M, and Simon H (1989) Experimental approach to individual vulnerability to psychostimulant addiction. Neurosci Biobehav Rev 13 141-147.

Di Chiara G and Imperato A (1988) Drugs abused by humans preferentially increase synaptic dopamine concentrations in the mesolimbic system of freely moving rats Proc Natl Acad Sci USA 85:5274-5278.

Dobbs LKK, Kaplan ARR, Lemos JCC, Matsui A, Rubinstein M, and Alvarez VAA (2016) Dopamine regulation of lateral inhibition between striatal neurons gates the stimulant actions of cocaine. Neuron 90:1100-1113.

Eban-Rothschild A, Rothschild G, Giardino WJ, Jones JR, and de Lecea L (2016) VTA dopaminergic neurons regulate ethologically relevant sleep-wake behaviors. Nat Neurosci 19:1356-1366.

Eldridge MAG, Lerchner W, Saunders RC, Kaneko H, Krausz KW, Gonzalez FJ, Ji B Higuchi M, Minamimoto T, and Richmond BJ (2016) Chemogenetic disconnection of monkey orbitofrontal and rhinal cortex reversibly disrupts reward value. Nat Neurosci 19:37-39.

Farrell MS, Pei Y, Wan Y, Yadav PN, Daigle TL, Urban DJ, Lee H-M, Sciaky N, Simmons A, Nonneman RJ, et al. (2013) A G $\alpha$ s DREADD mouse for selective modulation of cAMP production in striatopallidal neurons. Neuropsychopharmacology 38:854-862.

Ferguson SM, Eskenazi D, Ishikawa M, Wanat MJ, Phillips PEM, Dong Y, Roth BL, and Neumaier JF (2011) Transient neuronal inhibition reveals opposing roles of indirect and direct pathways in sensitization. Nat Neurosci 14:22-24.

Ferguson SM and Neumaier JF (2012) Grateful DREADDs: engineered receptors reveal how neural circuits regulate behavior. Neuropsychopharmacology $\mathbf{3 7}$ 296-297.

Ferguson SM, Phillips PEM, Roth BL, Wess J, and Neumaier JF (2013) Directpathway striatal neurons regulate the retention of decision-making strategies. J Neurosci 33:11668-11676.
Foldi CJ, Milton LK, and Oldfield BJ (2017) The role of mesolimbic reward neurocircuitry in prevention and rescue of the activity-based anorexia (ABA) phenotype in rats. Neuropsychopharmacology 42:2292-2300.

Fritz M, Klawonn AM, Nilsson A, Singh AK, Zajdel J, Wilhelms DB, Lazarus M, Löfberg A, Jaarola M, Kugelberg UÖ, et al. (2016) Prostaglandin-dependent modulation of dopaminergic neurotransmission elicits inflammation-induced aversion in mice. $J$ Clin Invest 126:695-705.

Gallo EF, Meszaros J, Sherman JD, Chohan MO, Teboul E, Choi CS, Moore H, Javitch JA, and Kellendonk C (2018) Accumbens dopamine D2 receptors increase motivation by decreasing inhibitory transmission to the ventral pallidum. Nat Commun 9:1086.

Garner AR, Rowland DC, Hwang SY, Baumgaertel K, Roth BL, Kentros C, and Mayford M (2012) Generation of a synthetic memory trace. Science 335: $1513-1516$

German CL, Baladi MG, McFadden LM, Hanson GR, and Fleckenstein AE (2015) Regulation of the dopamine and vesicular monoamine transporters: pharmacological targets and implications for disease. Pharmacol Rev 67:1005-1024.

Girasole AE, Lum MY, Nathaniel D, Bair-Marshall CJ, Guenthner CJ, Luo L, Kreitzer AC, and Nelson AB (2018) A subpopulation of striatal neurons mediates levodopa-induced dyskinesia. Neuron 97:787-795.e6.

Gomez JL, Bonaventura J, Lesniak W, Mathews WB, Sysa-Shah P, Rodriguez LA, Ellis RJ, Richie CT, Harvey BK, Dannals RF, et al. (2017) Chemogenetics revealed: DREADD occupancy and activation via converted clozapine. Science 357:503-507.

Gompf HS, Budygin EA, Fuller PM, and Bass CE (2015) Targeted genetic manipulations of neuronal subtypes using promoter-specific combinatorial AAVs in wild-type animals. Front Behav Neurosci 9:152.

Goto A, Nakahara I, Yamaguchi T, Kamioka Y, Sumiyama K, Matsuda M, Nakanishi S, and Funabiki K (2015) Circuit-dependent striatal PKA and ERK signaling underlies rapid behavioral shift in mating reaction of male mice. Proc Natl Acad Sci USA 112:6718-6723.

Grayson DS, Bliss-Moreau E, Machado CJ, Bennett J, Shen K, Grant KA, Fair DA, and Amaral DG (2016) The rhesus monkey connectome predicts disrupted functional networks resulting from pharmacogenetic inactivation of the amygdala. Neuron 91:453-466.

Guettier J-M, Gautam D, Scarselli M, Ruiz de Azua I, Li JH, Rosemond E, Ma X, Gonzalez FJ, Armbruster BN, Lu H, et al. (2009) A chemical-genetic approach to study G protein regulation of beta cell function in vivo. Proc Natl Acad Sci USA 106:19197-19202.

Heinsbroek JA, Neuhofer DN, Griffin WC III, Siegel GS, Bobadilla AC, Kupchik YM, and Kalivas PW (2017) Loss of plasticity in the D2-accumbens pallidal pathway promotes cocaine seeking. $J$ Neurosci 37:757-767.

Hnasko TS, Chuhma N, Zhang H, Goh GY, Sulzer D, Palmiter RD, Rayport S, and Edwards RH (2010) Vesicular glutamate transport promotes dopamine storage and glutamate corelease in vivo. Neuron 65:643-656

Hnasko TS, Perez FA, Scouras AD, Stoll EA, Gale SD, Luquet S, Phillips PEM, Kremer EJ, and Palmiter RD (2006) Cre recombinase-mediated restoration of nigrostriatal dopamine in dopamine-deficient mice reverses hypophagia and bradykinesia. Proc Natl Acad Sci USA 103:8858-8863.

Holmes A, Lachowicz JE, and Sibley DR (2004) Phenotypic analysis of dopamine receptor knockout mice; recent insights into the functional specificity of dopamine receptor subtypes. Neuropharmacology 47:1117-1134.

Hooks MS, Jones GH, Smith AD, Neill DB, and Justice JB Jr. (1991a) Individual differences in locomotor activity and sensitization. Pharmacol Biochem Behav 38: $467-470$.

Hooks MS, Jones GH, Smith AD, Neill DB, and Justice JB Jr. (1991b) Response to novelty predicts the locomotor and nucleus accumbens dopamine response to cocaine. Synapse 9:121-128.

Hornykiewicz O (1966) Dopamine (3-hydroxytyramine) and brain function. Pharmacol Rev 18:925-964.

Jackman SL, Beneduce BM, Drew IR, and Regehr WG (2014) Achieving highfrequency optical control of synaptic transmission. J Neurosci 34:7704-7714.

Jacobson KA, Gao ZG, Chen A, Barak D, Kim SA, Lee K, Link A, Rompaey PV, van Calenbergh S, and Liang BT (2001) Neoceptor concept based on molecular complementarity in GPCRs: a mutant adenosine A(3) receptor with selectively enhanced affinity for amine-modified nucleosides. J Med Chem 44:4125-4136.

Jann MW, Lam YW, and Chang WH (1994) Rapid formation of clozapine in guineapigs and man following clozapine-N-oxide administration. Arch Int Pharmacodyn Ther 328:243-250.

Jones SR, Gainetdinov RR, Jaber M, Giros B, Wightman RM, and Caron MG (1998) Profound neuronal plasticity in response to inactivation of the dopamine transporter. Proc Natl Acad Sci USA 95:4029-4034.

Jørgensen SH, Fitzpatrick CM, Gether U, Woldbye DPD, and Sørensen AT (2017) Chemogenetic modulation of $\mathrm{G}$ protein-coupled receptor signalling in visual attention research. Basic Clin Pharmacol Toxicol 121:373-381.

Kalivas PW (2008) Addiction as a pathology in prefrontal cortical regulation of corticostriatal habit circuitry. Neurotox Res 14:185-189.

Kawashima T, Okuno H, and Bito H (2014) A new era for functional labeling of neurons: activity-dependent promoters have come of age. Front Neural Circuits $\mathbf{8}$ 37.

Kerstetter KA, Wunsch AM, Nakata KG, Donckels E, Neumaier JF, and Ferguson SM (2016) Corticostriatal afferents modulate responsiveness to psychostimulant drugs and drug-associated stimuli. Neuropsychopharmacology 41:1128-1137.

Kim JI, Ganesan S, Luo SX, Wu YW, Park E, Huang EJ, Chen L, and Ding JB (2015) Aldehyde dehydrogenase 1a1 mediates a GABA synthesis pathway in midbrain dopaminergic neurons. Science 350:102-106.

Kobayashi T, Nishizawa D, Iwamura T, and Ikeda K (2007) Inhibition by cocaine of $\mathrm{G}$ protein-activated inwardly rectifying $\mathrm{K}+$ channels expressed in Xenopus oocytes. Toxicol In Vitro 21:656-664.

Koob GF and Le Moal M (1997) Drug abuse: hedonic homeostatic dysregulation. Science 278:52-58. 
Kristensen AS, Andersen J, Jørgensen TN, Sørensen L, Eriksen J, Loland CJ, Strømgaard K, and Gether U (2011) SLC6 neurotransmitter transporters: structure, function, and regulation. Pharmacol Rev 63:585-640.

Kumar S, Porcu P, Werner DF, Matthews DB, Diaz-Granados JL, Helfand RS, and Morrow AL (2009) The role of GABA(A) receptors in the acute and chronic effects of ethanol: a decade of progress. Psychopharmacology (Berl) 205:529-564.

Kupchik YM, Brown RM, Heinsbroek JA, Lobo MK, Schwartz DJ, and Kalivas PW (2015) Coding the direct/indirect pathways by D1 and D2 receptors is not valid for accumbens projections. Nat Neurosci 18:1230-1232.

Kuzhikandathil EV, Yu W, and Oxford GS (1998) Human dopamine D3 and D2L receptors couple to inward rectifier potassium channels in mammalian cell lines. Mol Cell Neurosci 12:390-402.

Lammel S, Hetzel A, Häckel O, Jones I, Liss B, and Roeper J (2008) Unique properties of mesoprefrontal neurons within a dual mesocorticolimbic dopamine system. Neuron 57:760-773.

Lammel S, Lim BK, and Malenka RC (2014) Reward and aversion in a heterogeneous midbrain dopamine system. Neuropharmacology 76 (Pt B):351-359.

Lammel S, Steinberg EE, Földy C, Wall NR, Beier K, Luo L, and Malenka RC (2015) Diversity of transgenic mouse models for selective targeting of midbrain dopamine neurons. Neuron 85:429-438.

Lavine N, Ethier N, Oak JN, Pei L, Liu F, Trieu P, Rebois RV, Bouvier M, Hebert TE and Van Tol HH (2002) G protein-coupled receptors form stable complexes with inwardly rectifying potassium channels and adenylyl cyclase. J Biol Chem $\mathbf{2 7 7}$ $46010-46019$

Lee JC, Wang LP, and Tsien JZ (2016) Dopamine rebound-excitation theory: putting brakes on PTSD. Front Psychiatry 7:163.

Lemos JC, Friend DM, Kaplan AR, Shin JH, Rubinstein M, Kravitz AV, and Alvarez VA (2016) Enhanced GABA transmission drives bradykinesia following loss of dopamine D2 receptor signaling. Neuron 90:824-838.

Li C, Sugam JA, Lowery-Gionta EG, McElligott ZA, McCall NM, Lopez AJ, McKlveen JM, Pleil KE, and Kash TL (2016) Mu opioid receptor modulation of dopamine neurons in the periaqueductal gray/dorsal raphe: a role in regulation of pain Neuropsychopharmacology 41:2122-2132.

Lindeberg J, Usoskin D, Bengtsson H, Gustafsson A, Kylberg A, Söderström S, and Ebendal T (2004) Transgenic expression of Cre recombinase from the tyrosine hydroxylase locus. Genesis 40:67-73.

Lindgren N, Usiello A, Goiny M, Haycock J, Erbs E, Greengard P, Hokfelt T, Borrell E, and Fisone G (2003) Distinct roles of dopamine D2L and D2S receptor isoforms in the regulation of protein phosphorylation at presynaptic and postsynaptic sites. Proc Natl Acad Sci USA 100:4305-4309.

Lobo MK and Nestler EJ (2011) The striatal balancing act in drug addiction: distinct roles of direct and indirect pathway medium spiny neurons. Front Neuroanat 5:41.

Locke TM, Soden ME, Miller SM, Hunker A, Knakal C, Licholai JA, Dhillon KS, Keene CD, Zweifel LS, and Carlson ES (2018) Dopamine D1 receptor-positive neurons in the lateral nucleus of the cerebellum contribute to cognitive behavior Biol Psychiatry 84:401-412.

Löffler S, Körber J, Nubbemeyer U, and Fehsel K (2012) Comment on "Impaired respiratory and body temperature control upon acute serotonergic neuron inhibition". Science 337:646-, author reply 646 .

Lopez-Huerta VG, Nakano Y, Bausenwein J, Jaidar O, Lazarus M, Cherassse Y, Garcia-Munoz M, and Arbuthnott G (2016) The neostriatum: two entities, one structure? Brain Struct Funct 221:1737-1749.

Luo Y-J, Li Y-D, Wang L, Yang S-R, Yuan X-S, Wang J, Cherasse Y, Lazarus M, Chen J-F, Qu W-M, et al. (2018) Nucleus accumbens controls wakefulness by a subpopulation of neurons expressing dopamine $\mathrm{D}_{1}$ receptors. Nat Commun 9:1576.

Maclaren DA, Browne RW, Shaw JK, Krishnan Radhakrishnan S, Khare P, España RA, and Clark SD (2016) Clozapine N-oxide administration produces behavioral effects in Long-Evans rats: implications for designing DREADD experiments. eNeuro 3 .

Mahler SV and Aston-Jones G (2018) CNO evil? considerations for the use of DREADDs in behavioral neuroscience. Neuropsychopharmacology 43:934-936.

Mahler SV, Vazey EM, Beckley JT, Keistler CR, McGlinchey EM, Kaufling J, Wilson SP, Deisseroth K, Woodward JJ, and Aston-Jones G (2014) Designer receptors show role for ventral pallidum input to ventral tegmental area in cocaine seeking. Nat Neurosci 17:577-585.

Manson A, Stirpe P, and Schrag A (2012) Levodopa-induced-dyskinesias clinical features, incidence, risk factors, management and impact on quality of life. J Parkinsons Dis 2:189-198.

Marchant NJ, Campbell EJ, Whitaker LR, Harvey BK, Kaganovsky K, Adhikary S, Hope BT, Heins RC, Prisinzano TE, Vardy E, et al. (2016a) Role of ventral subiculum in context-induced relapse to alcohol seeking after punishment-imposed abstinence. J Neurosci 36:3281-3294.

Marchant NJ, Whitaker LR, Bossert JM, Harvey BK, Hope BT, Kaganovsky K, Adhikary S, Prisinzano TE, Vardy E, Roth BL, et al. (2016b) Behavioral and physiological effects of a novel kappa-opioid receptor-based DREADD in rats. Neuropsychopharmacology 41:402-409.

Martin CK, Redman LM, Zhang J, Sanchez M, Anderson CM, Smith SR, and Ravussin E (2011) Lorcaserin, a 5-HT(2C) receptor agonist, reduces body weight by decreasing energy intake without influencing energy expenditure. $J$ Clin Endocrinol Metab 96:837-845.

Mayer D, Kahl E, Uzuneser TC, and Fendt M (2018) Role of the mesolimbic dopamine system in relief learning. Neuropsychopharmacology 43:1651-1659.

Michaelides M, Anderson SAR, Ananth M, Smirnov D, Thanos PK, Neumaier JF, Wang GJ, Volkow ND, and Hurd YL (2013) Whole-brain circuit dissection in freemoving animals reveals cell-specific mesocorticolimbic networks. J Clin Invest 123 $5342-5350$

Moehle MS, Pancani T, Byun N, Yohn SE, Wilson GH III, Dickerson JW, Remke DH Xiang Z, Niswender CM, Wess J, et al. (2017) Cholinergic projections to the substantia nigra pars reticulata inhibit dopamine modulation of basal ganglia through the M4 muscarinic receptor. Neuron 96:1358-1372.e4.
Moorman DE, James MH, McGlinchey EM, and Aston-Jones G (2015) Differential roles of medial prefrontal subregions in the regulation of drug seeking. Brain Res 1628 (Pt A):130-146.

Morales M and Margolis EB (2017) Ventral tegmental area: cellular heterogeneity, connectivity and behaviour. Nat Rev Neurosci 18:73-85.

Morales M and Root DH (2014) Glutamate neurons within the midbrain dopamine regions. Neuroscience 282:60-68.

Nair-Roberts RG, Chatelain-Badie SD, Benson E, White-Cooper H, Bolam JP, and Ungless MA (2008) Stereological estimates of dopaminergic, GABAergic and glutamatergic neurons in the ventral tegmental area, substantia nigra and retrorubral field in the rat. Neuroscience 152:1024-1031.

Nakajima K and Wess J (2012) Design and functional characterization of a novel, arrestin-biased designer G protein-coupled receptor. Mol Pharmacol 82:575-582. Napolitano F, Bonito-Oliva A, Federici M, Carta M, Errico F, Magara S, Martella G, Nisticò R, Centonze D, Pisani A, et al. (2010) Role of aberrant striatal dopamine D1 receptor/cAMP/protein kinase A/DARPP32 signaling in the paradoxical calming effect of amphetamine. $J$ Neurosci 30:11043-11056.

Neve KA, Seamans JK, and Trantham-Davidson H (2004) Dopamine receptor signaling $J$ Recept Signal Transduct Res 24:165-205.

Oishi Y, Suzuki Y, Takahashi K, Yonezawa T, Kanda T, Takata Y, Cherasse Y, and Lazarus M (2017a) Activation of ventral tegmental area dopamine neurons produces wakefulness through dopamine $\mathrm{D}_{2}$-like receptors in mice. Brain Struct Funct 222:2907-2915.

Oishi Y, Xu Q, Wang L, Zhang BJ, Takahashi K, Takata Y, Luo YJ, Cherasse Y, Schiffmann SN, de Kerchove d'Exaerde A, et al. (2017b) Slow-wave sleep is controlled by a subset of nucleus accumbens core neurons in mice. Nat Commun 8:734. Padgett CL, Lalive AL, Tan KR, Terunuma M, Munoz MB, Pangalos MN, MartínezHernández J, Watanabe M, Moss SJ, Luján R, et al. (2012) Methamphetamineevoked depression of $\mathrm{GABA}(\mathrm{B})$ receptor signaling in GABA neurons of the VTA. Neuron 73:978-989.

Padovan-Hernandez Y and Knackstedt LA (2018) Dose-dependent reduction in cocaine-induced locomotion by clozapine-N-oxide in rats with a history of cocaine self-administration. Neurosci Lett 674:132-135.

Parker PRL, Lalive AL, and Kreitzer AC (2016) Pathway-specific remodeling of thalamostriatal synapses in Parkinsonian mice. Neuron 89:734-740.

Pascoli V, Terrier J, Hiver A, and Lüscher C (2015) Sufficiency of mesolimbic dopamine neuron stimulation for the progression to addiction. Neuron 88:1054-1066.

Patriarchi T, Cho JR, Merten K, Howe MW, Marley A, Xiong WH, Folk RW, Broussard GJ, Liang R, Jang MJ, et al. (2018) Ultrafast neuronal imaging of dopamine dynamics with designed genetically encoded sensors. Science 360: eaat4422.

Patterson CM, Wong JM, Leinninger GM, Allison MB, Mabrouk OS, Kasper CL, Gonzalez IE, Mackenzie A, Jones JC, Kennedy RT, et al. (2015) Ventral tegmental area neurotensin signaling links the lateral hypothalamus to locomotor activity and striatal dopamine efflux in male mice. Endocrinology 156:1692-1700.

Peters J, Kalivas PW, and Quirk GJ (2009) Extinction circuits for fear and addiction overlap in prefrontal cortex. Learn Mem 16:279-288.

Piazza PV, Deminière JM, Le Moal M, and Simon H (1989) Factors that predict individual vulnerability to amphetamine self-administration. Science $\mathbf{2 4 5}$ : 1511-1513

Piazza PV, Deroche-Gamonent V, Rouge-Pont F, and Le Moal M (2000) Vertical shifts in self-administration dose-response functions predict a drug-vulnerable phenotype predisposed to addiction. J Neurosci 20:4226-4232.

Piazza PV, Rougé-Pont F, Deminière JM, Kharoubi M, Le Moal M, and Simon H (1991) Dopaminergic activity is reduced in the prefrontal cortex and increased in the nucleus accumbens of rats predisposed to develop amphetamine self-administration. Brain Res 567:169-174.

Pijnenburg AJ, Honig WM, Van der Heyden JA, and Van Rossum JM (1976) Effects of chemical stimulation of the mesolimbic dopamine system upon locomotor activity. Eur $J$ Pharmacol 35:45-58.

Pina MM and Cunningham CL (2017) Ethanol-seeking behavior is expressed directly through an extended amygdala to midbrain neural circuit. Neurobiol Learn Mem 137:83-91.

Pina MM, Young EA, Ryabinin AE, and Cunningham CL (2015) The bed nucleus of the stria terminalis regulates ethanol-seeking behavior in mice. Neuropharmacology 99:627-638.

Poulin JF, Caronia G, Hofer C, Cui Q, Helm B, Ramakrishnan C, Chan CS, Dombeck DA, Deisseroth K, and Awatramani R (2018) Mapping projections of molecularly defined dopamine neuron subtypes using intersectional genetic approaches. Nat Neurosci 21:1260-1271.

Prasad AA and McNally GP (2016) Ventral pallidum output pathways in contextinduced reinstatement of alcohol seeking. $J$ Neurosci 36:11716-11726.

Purohit K, Parekh PK, Kern J, Logan RW, Liu Z, Huang Y, McClung CA, Crabbe JC and Ozburn AR (2018) Pharmacogenetic manipulation of the nucleus accumbens alters binge-like alcohol drinking in mice. Alcohol Clin Exp Res 42:879-888.

Qualls-Creekmore E, Yu S, Francois M, Hoang J, Huesing C, Bruce-Keller A, Burk D, Berthoud HR, Morrison CD, and Münzberg H (2017) Galanin-expressing GABA neurons in the lateral hypothalamus modulate food reward and noncompulsive locomotion. J Neurosci 37:6053-6065.

Richardson NR and Roberts DC (1996) Progressive ratio schedules in drug self-administration studies in rats: a method to evaluate reinforcing efficacy. $J$ Neurosci Methods 66:1-11.

Richfield EK, Young AB, and Penney JB (1987) Comparative distribution of dopamine D-1 and D-2 receptors in the basal ganglia of turtles, pigeons, rats, cats, and monkeys. J Comp Neurol 262:446-463.

Robbins TW (2002) The 5-choice serial reaction time task: behavioural pharmacology and functional neurochemistry. Psychopharmacology (Berl) 163:362-380.

Robinson SE and Sohal VS (2017) Dopamine D2 receptors modulate pyramidal neurons in mouse medial prefrontal cortex through a stimulatory G-protein pathway. $J$ Neurosci 37:10063-10073. 
Roelofs TJM, Verharen JPH, van Tilborg GAF, Boekhoudt L, van der Toorn A, de Jong JW, Luijendijk MCM, Otte WM, Adan RAH, and Dijkhuizen RM (2017) A novel approach to map induced activation of neuronal networks using chemogenetics and functional neuroimaging in rats: a proof-of-concept study on the mesocorticolimbic system. Neuroimage 156:109-118.

Roman CW, Derkach VA, and Palmiter RD (2016) Genetically and functionally defined NTS to PBN brain circuits mediating anorexia. Nat Commun 7:11905.

Roth BL (2016) DREADDs for neuroscientists. Neuron 89:683-694.

Roy DS, Arons A, Mitchell TI, Pignatelli M, Ryan TJ, and Tonegawa S (2016) Memory retrieval by activating engram cells in mouse models of early Alzheimer's disease. Nature 531:508-512.

Runegaard AH, Jensen KL, Dencker D, Wörtwein G, and Gether U (2017a) Subjective perception of cocaine reward in mice assessed by a single exposure place preference (sePP) paradigm. J Neurosci Methods 289:85-92.

Runegaard AH, Jensen KL, Fitzpatrick CM, Dencker D, Weikop P, Gether U, and Rickhag M (2017b) Preserved dopaminergic homeostasis and dopaminerelated behaviour in hemizygous TH-Cre mice. Eur J Neurosci 45:121-128.

Runegaard AH, Sørensen AT, Fitzpatrick CM, Jørgensen SH, Petersen AV, Hansen NW, Weikop P, Andreasen JT, Mikkelsen JD, Perrier JF, et al. (2018) Locomotorand reward-enhancing effects of cocaine are differentially regulated by chemogenetic stimulation of Gi-signaling in dopaminergic neurons. eNeuro 5.

Salamone JD, Correa M, Nunes EJ, Randall PA, and Pardo M (2012) The behavioral pharmacology of effort-related choice behavior: dopamine, adenosine and beyond $J$ Exp Anal Behav 97:125-146.

Sandhu EC, Fernando ABP, Irvine EE, Tossell K, Kokkinou M, Glegola J, Smith MA Howes OD, Withers DJ, and Ungless MA (2018) Phasic stimulation of midbrain dopamine neuron activity reduces salt consumption. eNeuro 5:ENEURO.0064-18.2018.

Savitt JM, Jang SS, Mu W, Dawson VL, and Dawson TM (2005) Bcl-x is required for proper development of the mouse substantia nigra. J Neurosci 25:6721-6728.

Schultz W (2007) Multiple dopamine functions at different time courses. Annu Rev Neurosci 30:259-288.

Schwarz LA, Miyamichi K, Gao XJ, Beier KT, Weissbourd B, DeLoach KE, Ren J, Ibanes S, Malenka RC, Kremer EJ, et al. (2015) Viral-genetic tracing of the inputoutput organization of a central noradrenaline circuit. Nature 524:88-92.

Scofield MD, Boger HA, Smith RJ, Li H, Haydon PG, and Kalivas PW (2015) GqDREADD selectively initiates glial glutamate release and inhibits cue-induced cocaine seeking. Biol Psychiatry 78:441-451.

Sharpe AL, Varela E, Bettinger L, and Beckstead MJ (2014) Methamphetamine selfadministration in mice decreases GIRK channel-mediated currents in midbrain dopamine neurons. Int $J$ Neuropsychopharmacol 18:1-10.

Smith KS, Bucci DJ, Luikart BW, and Mahler SV (2016) DREADDS: use and application in behavioral neuroscience. Behav Neurosci 130:137-155.

Söderpalm B and Ericson M (2013) Neurocircuitry involved in the development of alcohol addiction: the dopamine system and its access points. Curr Top Behav Neurosci 13:127-161.

Stamatakis AM, Jennings JH, Ung RL, Blair GA, Weinberg RJ, Neve RL, Boyce F, Mattis J, Ramakrishnan C, Deisseroth K, et al. (2013) A unique population of ventral tegmental area neurons inhibits the lateral habenula to promote reward. Neuron 80:1039-1053.

Stauffer WR, Lak A, Yang A, Borel M, Paulsen O, Boyden ES, and Schultz W (2016) Dopamine neuron-specific optogenetic stimulation in rhesus macaques. Cell $\mathbf{1 6 6}$ 1564-1571.e6.

Strader CD, Gaffney T, Sugg EE, Candelore MR, Keys R, Patchett AA, and Dixon RA (1991) Allele-specific activation of genetically engineered receptors. J Biol Chem 266:5-8.

Stuber GD, Stamatakis AM, and Kantak PA (2015) Considerations when using credriver rodent lines for studying ventral tegmental area circuitry. Neuron $\mathbf{8 5}$ 439-445.

Stuber GD, Hnasko TS, Britt JP, Edwards RH, and Bonci A (2010) Dopaminergic terminals in the nucleus accumbens but not the dorsal striatum corelease glutamate. J Neurosci 30:8229-8233.

Sulzer D (2011) How addictive drugs disrupt presynaptic dopamine neurotransmission. Neuron 69:628-649.

Sun F, Zeng J, Jing M, Zhou J, Feng J, Owen SF, Luo Y, Li F, Wang H, Yamaguchi T, et al. (2018) A genetically encoded fluorescent sensor enables rapid and specific detection of dopamine in flies, fish, and mice. Cell 174:481-496.e19.

Sun HX, Wang DR, Ye CB, Hu ZZ, Wang CY, Huang ZL, and Yang SR (2017) Activation of the ventral tegmental area increased wakefulness in mice. Sleep Biol Rhythms 15:107-115.

Svoboda K and Yasuda R (2006) Principles of two-photon excitation microscopy and its applications to neuroscience. Neuron 50:823-839.

Tervo DGR, Hwang B-Y, Viswanathan S, Gaj T, Lavzin M, Ritola KD, Lindo S, Michael S, Kuleshova E, Ojala D, et al. (2016) A designer AAV variant permits efficient retrograde access to projection neurons. Neuron 92:372-382.
Todd TP, Vurbic D, and Bouton ME (2014) Behavioral and neurobiological mechanisms of extinction in Pavlovian and instrumental learning. Neurobiol Learn Mem 108:52-64.

Torres GE and Amara SG (2007) Glutamate and monoamine transporters: new visions of form and function. Curr Opin Neurobiol 17:304-312.

Tritsch NX, Oh WJ, Gu C, and Sabatini BL (2014) Midbrain dopamine neurons sustain inhibitory transmission using plasma membrane uptake of GABA, not synthesis. eLife 3:e01936.

Tritsch NX and Sabatini BL (2012) Dopaminergic modulation of synaptic transmission in cortex and striatum. Neuron 76:33-50.

Ungerstedt U and Arbuthnott GW (1970) Quantitative recording of rotational behavior in rats after 6-hydroxy-dopamine lesions of the nigrostriatal dopamine system. Brain Res 24:485-493.

Urban DJ and Roth BL (2015) DREADDs (designer receptors exclusively activated by designer drugs): chemogenetic tools with therapeutic utility. Annu Rev Pharmacol Toxicol 55:399-417.

Valencia-Torres L, Olarte-Sánchez CM, Lyons DJ, Georgescu T, Greenwald-Yarnell M, Myers MG Jr., Bradshaw CM, and Heisler LK (2017) Activation of ventral tegmental area 5-HT2C receptors reduces incentive motivation. Neuropsychopharmacology 42:1511-1521.

Vardy E, Robinson JE, Li C, Olsen RHJ, DiBerto JF, Giguere PM, Sassano FM, Huang XP, Zhu H, Urban DJ, et al. (2015) A new DREADD facilitates the multiplexed chemogenetic interrogation of behavior. Neuron 86:936-946.

Vazey EM and Aston-Jones G (2013) New tricks for old dogmas: optogenetic and designer receptor insights for Parkinson's disease. Brain Res 1511:153-163.

Vengeliene V, Bilbao A, Molander A, and Spanagel R (2008) Neuropharmacology of alcohol addiction. Br J Pharmacol 154:299-315.

Volkow ND, Fowler JS, Wang GJ, and Swanson JM (2004) Dopamine in drug abuse and addiction: results from imaging studies and treatment implications. Mol Psychiatry 9:557-569.

Volkow ND, Fowler JS, Wang G, Swanson JM, and Telang F (2007) Dopamine in drug abuse and addiction: results of imaging studies and treatment implications. Arch Neurol 64:1575-1579.

Wakaizumi K, Kondo T, Hamada Y, Narita M, Kawabe R, Narita H, Watanabe M, Kato S, Senba E, Kobayashi K, et al. (2016) Involvement of mesolimbic dopaminergic network in neuropathic pain relief by treadmill exercise: a study for specific neural control with Gi-DREADD in mice. Mol Pain 12:1-11.

Wang H, La Russa M, and Qi LS (2016) CRISPR/Cas9 in genome editing and beyond Annu Rev Biochem 85:227-264.

Wang S, Tan Y, Zhang JE, and Luo M (2013) Pharmacogenetic activation of midbrain dopaminergic neurons induces hyperactivity. Neurosci Bull 29:517-524.

Wang X-F, Liu J-J, Xia J, Liu J, Mirabella V, and Pang ZP (2015) Endogenous glucagon-like peptide-1 suppresses high-fat food intake by reducing synaptic drive onto mesolimbic dopamine neurons. Cell Reports 12:726-733.

Wasserman DI, Tan JMJ, Kim JC, and Yeomans JS (2016) Muscarinic control of rostromedial tegmental nucleus GABA neurons and morphine-induced locomotion. Eur J Neurosci 44:1761-1770.

Wayman WN and Woodward JJ (2018) Chemogenetic excitation of accumbensprojecting infralimbic cortical neurons blocks toluene-induced conditioned place preference. J Neurosci 38:1462-1471.

Welter M, Vallone D, Samad TA, Meziane H, Usiello A, and Borrelli E (2007) Absence of dopamine D2 receptors unmasks an inhibitory control over the brain circuitries activated by cocaine. Proc Natl Acad Sci USA 104:6840-6845.

Whissell PD, Tohyama S, and Martin LJ (2016) The use of DREADDs to deconstruct behavior. Front Genet 7:70.

Wise RA and Bozarth MA (1985) Brain mechanisms of drug reward and euphoria. Psychiatr Med 3:445-460.

Wunsch AM, Yager LM, Donckels EA, Le CT, Neumaier JF, and Ferguson SM (2017) Chemogenetic inhibition reveals midline thalamic nuclei and thalamo-accumbens projections mediate cocaine-seeking in rats. Eur J Neurosci 46:1850-1862.

Xu P, He Y, Cao X, Valencia-Torres L, Yan X, Saito K, Wang C, Yang Y, Hinton A Jr. Zhu L, et al. (2017) Activation of serotonin 2C receptors in dopamine neurons inhibits binge-like eating in mice. Biol Psychiatry 81:737-747.

Yan Z, Song WJ, and Surmeier J (1997) D2 dopamine receptors reduce N-type Ca2+ currents in rat neostriatal cholinergic interneurons through a membranedelimited, protein-kinase-C-insensitive pathway. $J$ Neurophysiol 77:1003-1015.

Zhong P, Liu X, Zhang Z, Hu Y, Liu SJ, Lezama-Ruiz M, Joksimovic M, and Liu Q-S (2014) Cyclin-dependent kinase 5 in the ventral tegmental area regulates depression-related behaviors. $J$ Neurosci 34:6352-6366.

Zhou FM, Liang Y, Salas R, Zhang L, De Biasi M, and Dani JA (2005) Corelease of dopamine and serotonin from striatal dopamine terminals. Neuron 46:65-74.

Zhu X, Ottenheimer D, and DiLeone RJ (2016) Activity of D1/2 receptor expressing neurons in the nucleus accumbens regulates running, locomotion, and food intake. Front Behav Neurosci 10:66. 